Article

\title{
Some Dynamic Hilbert-Type Inequalities on Time Scales
}

\author{
Ghada AlNemer ${ }^{1}\left(\mathbb{D}\right.$, Mohammed Zakarya ${ }^{2,3, *}$, Hoda A. Abd El-Hamid ${ }^{4}$, Praveen Agarwal ${ }^{5}$ \\ and Haytham M. Rezk ${ }^{6}$ (D) \\ 1 Department of Mathematical Science, College of Science, Princess Nourah bint Abdulrahman University, \\ P.O. Box 105862, Riyadh 11656, Saudi Arabia; gnnemer@pnu.edu.sa \\ 2 Department of Mathematics, College of Science, King Khalid University, P.O. Box 9004, \\ Abha 61413, Saudi Arabia \\ 3 Department of Mathematics, Faculty of Science, Al-Azhar University, Assiut 71524, Egypt \\ 4 Department of Mathematics and Computer Science, Faculty of Science, Beni-Suef University, \\ Beni-Suef 62511, Egypt; Hoda.Ali@science.bsu.edu.eg \\ 5 Department of Mathematics, Anand International College of Engineering, Jaipur 303012, Rajasthan, India; \\ praveen.agarwal@anandice.ac.in \\ 6 Department of Mathematics, Faculty of Science, Al-Azhar University, Nasr City 11884, Egypt; \\ haythamrezk@azhar.edu.eg \\ * Correspondence: mzibrahim@kku.edu.sa
}

Received: 25 July 2020; Accepted: 16 August 2020; Published: 25 August 2020

check for updates

\begin{abstract}
Throughout this article, we will demonstrate some new generalizations of dynamic Hilbert type inequalities, which are used in various problems involving symmetry. We develop a number of those symmetric inequalities to a general time scale. From these inequalities, as particular cases, we formulate some integral and discrete inequalities that have been demonstrated in the literature and also extend some of the dynamic inequalities that have been achieved in time scales.
\end{abstract}

Keywords: Hilbert's inequality; Hölder's inequality; Jensen's inequality; time scales

MSC: 26D15; 34A40; 39A12; 34N05

\section{Introduction}

In recent years, Hilbert's double-series inequality and its integral version [1] (pp. 253-254) has been granted significant attention by many scholars (see, for example, in [2-12]). In particular, B. G. Pachpatte [13] defined a new inequalities close to that of Hilbert as follows. Let $a_{s}: N_{p}=\{0,1,2, \ldots, p\} \subset \mathbb{N} \rightarrow \mathbb{R}$ and $b_{\vartheta}: N_{q}=\{0,1,2, \ldots, q\} \subset \mathbb{N} \rightarrow \mathbb{R}$ for $p, q \in \mathbb{N}$ and $a(0)=b(0)=0$. Then

$$
\begin{aligned}
\sum_{s=1}^{p} \sum_{\vartheta=1}^{q} \frac{\left|a_{s}\right|\left|b_{\vartheta}\right|}{s+\vartheta} \leq & C(p, q)\left(\sum_{s=1}^{p}(p-s+1)\left|\nabla a_{s}\right|^{2}\right)^{\frac{1}{2}} \\
& \times\left(\sum_{\vartheta=1}^{q}(q-\vartheta+1)\left|\nabla b_{\vartheta}\right|^{2}\right)^{\frac{1}{2}}
\end{aligned}
$$

where $\nabla a_{s}=a_{s}-a_{s-1}, \nabla b_{\vartheta}=b_{\vartheta}-b_{\vartheta-1}$ and

$$
C(p, q)=\frac{1}{2} \sqrt{p q}
$$


An integral version of (1) is established in the next consequence. Let $f(s)$ and $g(\vartheta)$ be real-valued continuous functions defined on $I_{x}=[0, \infty) \subset \mathbb{R}$ and $I_{y}=[0, \infty) \subset \mathbb{R}$ for $x, y \in I_{0}=(0, \infty) \subset \mathbb{R}$, respectively, and $f(0)=g(0)=0$. Then

$$
\begin{aligned}
\int_{0}^{x} \int_{0}^{y} \frac{|f(s)||g(\vartheta)|}{s+\vartheta} d s d \vartheta \leq & C^{*}(x, y)\left(\int_{0}^{x}(x-s)\left|f^{\prime}(s)\right|^{2} d s\right)^{\frac{1}{2}} \\
& \times\left(\int_{0}^{y}(y-\vartheta)\left|g^{\prime}(\vartheta)\right|^{2} d \vartheta\right)^{\frac{1}{2}}
\end{aligned}
$$

where $u^{\prime}(t)$ denote the usual derivative of function $u(t)$ and

$$
C^{*}(x, y)=\frac{1}{2} \sqrt{x y}
$$

In [14], Pachpatte gave some generalizations of (1) and (2) as follows. Let $\lambda, \mu>1$ be constants such that $1 / \lambda+1 / \mu=1$. If $a_{s}: N_{p}=\{0,1,2, \ldots, p\} \subset \mathbb{N} \rightarrow \mathbb{R}$ and $b_{\vartheta}: N_{q}=\{0,1,2, \ldots, q\} \subset \mathbb{N} \rightarrow \mathbb{R}$ for $p, q \in \mathbb{N}$ and $a(0)=b(0)=0$, then

$$
\begin{aligned}
\sum_{s=1}^{p} \sum_{\vartheta=1}^{q} \frac{\left|a_{s}\right|\left|b_{\vartheta}\right|}{\mu s^{\lambda-1}+\lambda \vartheta^{\mu-1}} \leq & C^{* *}(\lambda, \mu)\left(\sum_{s=1}^{p}(p-s+1)\left|\nabla a_{s}\right|^{\lambda}\right)^{\frac{1}{\lambda}} \\
& \times\left(\sum_{\vartheta=1}^{q}(q-\vartheta+1)\left|\nabla b_{\vartheta}\right|^{\mu}\right)^{\frac{1}{\mu}},
\end{aligned}
$$

where $\nabla a_{S}=a_{s}-a_{S-1}, \nabla b_{\vartheta}=b_{\vartheta}-b_{\vartheta-1}$ and

$$
C^{* *}(\lambda, \mu)=\frac{1}{\lambda \mu}(p)^{\frac{\lambda-1}{\lambda}}(q)^{\frac{\mu-1}{\mu}} .
$$

An integral version of (3) is established in the next consequence. Let $\lambda, \mu>1$ be constants such that $1 / \lambda+1 / \mu=1$. If $f(s)$ and $g(\vartheta)$ are real-valued continuous functions defined on $I_{x}=[0, \infty) \subset \mathbb{R}$ and $I_{y}=[0, \infty) \subset \mathbb{R}$ for $x, y \in I_{0}=(0, \infty) \subset \mathbb{R}$, respectively, and $f(0)=g(0)=0$, then

$$
\begin{aligned}
\int_{0}^{x} \int_{0}^{y} \frac{|f(s)||g(\vartheta)|}{\mu s^{\lambda-1}+\lambda \vartheta^{\mu-1}} d s d \vartheta \leq & D(\lambda, \mu)\left(\int_{0}^{x}(x-s)\left|f^{\prime}(s)\right|^{\lambda} d s\right)^{\frac{1}{\lambda}} \\
& \times\left(\int_{0}^{y}(y-\vartheta)\left|g^{\prime}(\vartheta)\right|^{\mu} d \vartheta\right)^{\frac{1}{\mu}}
\end{aligned}
$$

where

$$
D(\lambda, \mu)=\frac{1}{\lambda \mu}(x)^{\frac{\lambda-1}{\lambda}}(y)^{\frac{\mu-1}{\mu}}
$$

In [15], the authors gave another generalization of (3) and (4) as follows. Let $\lambda, \mu>1$ be constants such that $1 / \lambda+1 / \mu=1$. If $a_{s}: N_{p}=\{0,1,2, \ldots, p\} \subset \mathbb{N} \rightarrow \mathbb{R}$ and $b_{\vartheta}: N_{q}=\{0,1,2, \ldots, q\} \subset \mathbb{N} \rightarrow \mathbb{R}$ for $p, q \in \mathbb{N}$ and $a(0)=b(0)=0$, then

$$
\begin{aligned}
\sum_{s=1}^{p} \sum_{\vartheta=1}^{q} \frac{\left|a_{s}\right|\left|b_{t}\right|}{\mu s^{\frac{(\lambda-1)(\lambda+\mu)}{\lambda \mu}}+\lambda \vartheta^{\frac{(\mu-1)(\lambda+\mu)}{\lambda \mu}}} \leq & D^{*}(\lambda, \mu)\left(\sum_{s=1}^{p}(p-s+1)\left|\nabla a_{s}\right|^{\lambda}\right)^{\frac{1}{\lambda}} \\
& \times\left(\sum_{\vartheta=1}^{q}(q-\vartheta+1)\left|\nabla b_{\vartheta}\right|^{\mu}\right)^{\frac{1}{\mu}},
\end{aligned}
$$


where $\nabla a_{s}=a_{s}-a_{s-1}, \nabla b_{\vartheta}=b_{\vartheta}-b_{\vartheta-1}$ and

$$
D^{*}(\lambda, \mu)=\frac{1}{\lambda+\mu}(p)^{\frac{\lambda-1}{\lambda}}(q)^{\frac{\mu-1}{\mu}} .
$$

An integral analogue of (5) is established in the next consequence. Let $\lambda, \mu>1$ be constants such that $1 / \lambda+1 / \mu=1$ and $f(s) \in C^{1}\left[[0, x), \mathbb{R}^{+}\right], g(\vartheta) \in C^{1}\left[[0, y), \mathbb{R}^{+}\right]$with $f(0)=g(0)=0$. Then

$$
\begin{aligned}
\int_{0}^{x} \int_{0}^{y} \frac{|f(s)||g(\vartheta)|}{\mu s \frac{(\lambda-1)(\lambda+\mu)}{\lambda \mu}+\lambda \vartheta \frac{(\mu-1)(\lambda+\mu)}{\lambda \mu}} d s d \vartheta \leq & D^{* *}(\lambda, \mu)\left(\int_{0}^{x}(x-s)\left|f^{\prime}(s)\right|^{\lambda} d s\right)^{\frac{1}{\lambda}} \\
& \times\left(\int_{0}^{y}(y-\vartheta)\left|g^{\prime}(\vartheta)\right|^{\mu} d \vartheta\right)^{\frac{1}{\mu}},
\end{aligned}
$$

where

$$
D^{* *}(\lambda, \mu)=\frac{1}{\lambda+\mu}(x)^{\frac{\lambda-1}{\lambda}}(y)^{\frac{\mu-1}{\mu}} .
$$

On the other hand, Hilger [16] suggested the theory of time scales to unify discrete and continuous analysis, based on which some authors have studied the Hilbert-kind inequalities on time scales (see in [17-21]). In the following, the time scale $\mathbb{T}$ is a non-empty closed subset of $\mathbb{R}$ and defines the time scale interval $[k, l]_{\mathbb{T}}$ by

$$
[k, l]_{\mathbb{T}}=[k, l] \cap \mathbb{T} .
$$

Let $\mathrm{C}_{r d}$ denotes the set of right-dense continuous (rd-continuous), $\mathrm{CC}_{r d}$ denotes the set of functions $g\left(\vartheta_{1}, \vartheta_{2}\right)$ on $\mathbb{T}_{1} \times \mathbb{T}_{2}$ where $g$ is rd-continuous in $\vartheta_{1}$ and $\vartheta_{2}$ and $C_{r d}^{1}$ denotes the set of all functions $\mathrm{CC}_{r d}$ for which both the $\Delta_{1}$ partial derivative and $\Delta_{2}$ partial derivative exists and are in $\mathrm{CC}_{r d}$. For details on calculating the time scales see in [22,23].

The following useful relationships are often used between the time scale calculus $\mathbb{T}$ and the difference calculus $\mathbb{R}$ and the difference calculus $\mathbb{Z}$. Please mind that

(i) if $\mathbb{T}=\mathbb{R}$, then

$$
\sigma(\vartheta)=\vartheta, f^{\Delta}(\vartheta)=f^{\prime}(\vartheta), \int_{k}^{l} f^{\Delta}(\vartheta) \Delta \vartheta=\int_{k}^{l} f(\vartheta) d \vartheta
$$

(ii) if $\mathbb{T}=\mathbb{Z}$, then

$$
\sigma(\vartheta)=\vartheta+1, f^{\Delta}(\vartheta)=\Delta f(\vartheta), \int_{k}^{l} f^{\Delta}(\vartheta) \Delta \vartheta=\sum_{\vartheta=k}^{l-1} f(\vartheta)
$$

Within the following, we display some basic lemmas and some algebraic inequalities that play a key role in inaugurating the major findings of this paper.

Lemma 1 (Hölder's inequality in one dimension [24]). Let $k, l \in \mathbb{T}$ and $\zeta, \chi \in \mathrm{C}_{r d}(\mathbb{T}, \mathbb{R})$. Then

$$
\int_{k}^{l}|\zeta(\vartheta) \chi(\vartheta)| \Delta \vartheta \leq\left(\int_{k}^{l}|\zeta(\vartheta)|^{\lambda} \Delta \vartheta\right)^{\frac{1}{\lambda}}\left(\int_{k}^{l}|\chi(\vartheta)|^{\mu} \Delta \vartheta\right)^{\frac{1}{\mu}}
$$

where $\lambda, \mu>1$ and $1 / \lambda+1 / \mu=1$.

Lemma 2 (Hölder's inequality in two dimensions [24]). Let $k, l \in \mathbb{T}$ and $\zeta, \chi \in \mathrm{CC}_{r d}^{1}\left([k, l]_{\mathbb{T}} \times[k, l]_{\mathbb{T}}\right.$, $\mathbb{R})$. Then,

$$
\int_{k}^{l} \int_{k}^{l}|\zeta(s, \vartheta) \chi(s, \vartheta)| \Delta s \Delta \vartheta \leq\left(\int_{k}^{l} \int_{k}^{l}|\zeta(s, \vartheta)|^{\lambda} \Delta s \Delta \vartheta\right)^{\frac{1}{\lambda}}\left(\int_{k}^{l} \int_{k}^{l}|\chi(s, \vartheta)|^{\mu} \Delta s \Delta \vartheta\right)^{\frac{1}{\mu}},
$$

where $\lambda>1$ and $\mu=1 /(\lambda-1)$. 
Lemma 3 (Jensen's inequality in one dimension [24]). Let $k, l \in \mathbb{T}$ and $m, n \in \mathbb{R}$. Assume that $\zeta \in \mathrm{C}_{r d}\left([k, l]_{\mathbb{T}},(m, n)\right)$ and $\chi \in \mathrm{C}_{r d}\left([a, b]_{\mathbb{T}}, \mathbb{R}\right)$ are non-negative with $\int_{k}^{l}|\zeta(\vartheta)| \Delta \vartheta>0$. If $\Theta \in \mathrm{C}((m, n)$, $\mathbb{R})$ ba a convex function, then

$$
\Theta\left(\frac{\int_{k}^{l}|\zeta(\vartheta)| \chi(\vartheta) \Delta \vartheta}{\int_{k}^{l}|\zeta(\vartheta)| \Delta \vartheta}\right) \leq \frac{\int_{k}^{l}|\zeta(\vartheta)| \Theta(\chi(\vartheta)) \Delta \vartheta}{\int_{k}^{l}|\zeta(\vartheta)| \Delta \vartheta} .
$$

Lemma 4 (Jensen's inequality in two dimensions [25] Theorem 3.1). Let $s, \vartheta \in \mathrm{R}$ and $-\infty \leq p<q \leq \infty$. If $\zeta \in \mathrm{CC}_{r d}^{1}(\mathrm{R},(p, q))$ and $\Theta:(p, q) \rightarrow \mathbb{R}$ be a convex function, then

$$
\Theta\left(\frac{\int_{k}^{l} \int_{k}^{l} \zeta(s, \vartheta) \Delta_{1} s \Delta_{2} \vartheta}{\int_{k}^{l} \int_{k}^{l} \Delta_{1} s \Delta_{2} \vartheta}\right) \leq \frac{\int_{k}^{l} \int_{k}^{l} \Theta(\zeta(s, \vartheta)) \Delta_{1} s \Delta_{2} \vartheta}{\int_{k}^{l} \int_{k}^{l} \Delta_{1} s \Delta_{2} \vartheta},
$$

where $\mathrm{R}$ is a rectangle in $\mathbb{T}_{1} \times \mathbb{T}_{2}$ defined by

$$
\mathrm{R}=[k, l) \times[m, n)=\{(s, \vartheta): s \in[k, l), \vartheta \in[m, n)\} .
$$

Lemma 5 (Fubini's theorem [26] Theorem 1.1). Let $\mathbb{T}_{1}$ and $\mathbb{T}_{2}$ be two time scales. Suppose that $\zeta$ : $\mathbb{T}_{1} \times \mathbb{T}_{2} \rightarrow \mathbb{R}$ is a $\Delta$-integrable function with respect to both time scales. Define

$$
\Omega(\vartheta)=\int_{\mathbb{T}_{1}} \zeta(s, \vartheta) \Delta s, \quad \vartheta \in \mathbb{T}_{2}
$$

and

$$
\Pi(s)=\int_{\mathbb{T}_{2}} \zeta(s, \vartheta) \Delta \vartheta, \quad s \in \mathbb{T}_{1} .
$$

Then, $\Omega$ is $\Delta$-integrable on $\mathbb{T}_{2}$ and $\Pi$ is $\Delta$-integrable on $\mathbb{T}_{1}$ and

$$
\int_{\mathbb{T}_{1}} \Delta s \int_{\mathbb{T}_{2}} \zeta(s, \vartheta) \Delta \vartheta=\int_{\mathbb{T}_{2}} \Delta \vartheta \int_{\mathbb{T}_{1}} \zeta(s, \vartheta) \Delta s
$$

Lemma 6 (Young's inequality [27]). Let $r>0, \mu_{q}>0$ and $\sum_{q=1}^{p} \mu_{q}=\Omega_{p}$. Then

$$
\left\{\prod_{q=1}^{p} s_{q}^{\mu_{q}}\right\}^{\frac{1}{\Omega_{p}}} \leq\left\{\frac{1}{\Omega_{p}} \sum_{q=1}^{p} \mu_{q} s_{q}^{r}\right\}^{\frac{1}{r}} .
$$

The symmetry index is the most important parameter when evaluating functional asymmetries in athletes of different disciplines. Hence, the first objective of this paper is to establish a new inequality symmetry to Hilbert's type inequality. Our findings provide new estimates on time-scale for this form of inequality. During that paper, we must assume that all functions found in the theorems statements are non-negative, right-dense continuous (rd-continuous) and that the integrals considered exist.

\section{Main Results}

In this section, we state and prove our main results. Namely, we set a time scale model for inequalities (5) and (6). To prove our next theorems, we will assume that $\lambda, \mu$ be any two real numbers such that $\lambda, \mu>1$ with $1 / \lambda+1 / \mu=1$. 


\subsection{The One Dimension Version}

Theorem 1. Let $s, \vartheta$ and $t_{0} \in \mathbb{T}, f(s) \in \mathrm{C}_{r d}^{1}\left(\left[t_{0}, x\right)_{\mathbb{T}}, \mathbb{R}^{+}\right), g(\vartheta) \in \mathrm{C}_{r d}^{1}\left(\left[t_{0}, y\right)_{\mathbb{T}}, \mathbb{R}^{+}\right)$and $f\left(t_{0}\right)=g\left(t_{0}\right)=0$. Then, for $s \in\left[t_{0}, x\right)_{\mathbb{T}}$ and $\vartheta \in\left[t_{0}, y\right)_{\mathbb{T}}$, we have

$$
\begin{aligned}
& \int_{t_{0}}^{x} \int_{t_{0}}^{y} \frac{|f(s)||g(\vartheta)|}{\mu\left(s-t_{0}\right)^{\frac{(\lambda-1)(\lambda+\mu)}{\lambda \mu}}+\lambda\left(\vartheta-t_{0}\right)^{\frac{(\mu-1)(\lambda+\mu)}{\lambda \mu}}} \Delta s \Delta \vartheta \\
\leq & E(\lambda, \mu)\left(\int_{t_{0}}^{x}(\sigma(x)-s)\left|f^{\Delta}(s)\right|^{\lambda} \Delta s\right)^{\frac{1}{\lambda}} \\
& \times\left(\int_{t_{0}}^{y}(\sigma(y)-\vartheta)\left|g^{\Delta}(\vartheta)\right|^{\mu} \Delta \vartheta\right)^{\frac{1}{\mu}}
\end{aligned}
$$

where

$$
E(\lambda, \mu)=\frac{1}{\lambda+\mu}\left(x-t_{0}\right)^{\frac{\lambda-1}{\lambda}}\left(y-t_{0}\right)^{\frac{\mu-1}{\mu}}
$$

for $x, y \in I_{0}=\left[t_{0}, \infty\right) \cap \mathbb{T}$.

Proof. From the hypotheses, we have the following two identities hold,

$$
\begin{aligned}
& |f(s)|=\int_{t_{0}}^{s}\left|f^{\Delta}(\tau)\right| \Delta \tau, \\
& |g(\vartheta)|=\int_{t_{0}}^{\vartheta}\left|g^{\Delta}(\xi)\right| \Delta \xi,
\end{aligned}
$$

for $s \in\left[t_{0}, x\right)_{\mathbb{T}}, t \in\left[t_{0}, y\right)_{\mathbb{T}}$. Further, by using Hölder's integral inequality (9), we have

$$
\begin{aligned}
& |f(s)| \leq\left(s-t_{0}\right)^{\frac{\lambda-1}{\lambda}}\left(\int_{t_{0}}^{s}\left|f^{\Delta}(\tau)\right|^{\lambda} \Delta \tau\right)^{\frac{1}{\lambda}}, \\
& |g(\vartheta)| \leq\left(\vartheta-t_{0}\right)^{\frac{\mu-1}{\mu}}\left(\int_{t_{0}}^{\vartheta}\left|g^{\Delta}(\xi)\right|^{\mu} \Delta \xi\right)^{\frac{1}{\mu}} .
\end{aligned}
$$

By multiplying (19) and (20), we get

$$
|f(s)||g(\vartheta)| \leq\left(s-t_{0}\right)^{\frac{\lambda-1}{\lambda}}\left(\vartheta-t_{0}\right)^{\frac{\mu-1}{\mu}}\left(\int_{t_{0}}^{s}\left|f^{\Delta}(\tau)\right|^{\lambda} \Delta \tau\right)^{\frac{1}{\lambda}}\left(\int_{t_{0}}^{\vartheta}\left|g^{\Delta}(\xi)\right|^{\mu} \Delta \xi\right)^{\frac{1}{\mu}} .
$$

Using the inequality (14), we note

$$
\left(s_{1}^{\omega_{1}} s_{2}^{\omega_{2}}\right)^{\frac{r}{\omega_{1}+\omega_{2}}} \leq \frac{1}{\omega_{1}+\omega_{2}}\left(\omega_{1} s_{1}^{r}+\omega_{2} s_{2}^{r}\right)
$$

Now, by setting $s_{1}=\left(s-t_{0}\right)^{\lambda-1}, s_{2}=\left(\vartheta-t_{0}\right)^{\mu-1}, \omega_{1}=1 / \lambda, \omega_{2}=1 / \mu$ and $r=\omega_{1}+\omega_{2}$ in (22), we get

$$
\left(s-t_{0}\right)^{\frac{\lambda-1}{\lambda}}\left(\vartheta-t_{0}\right)^{\frac{\mu-1}{\mu}} \leq \frac{\lambda \mu}{\lambda+\mu}\left(\frac{\left(s-t_{0}\right)^{\frac{(\lambda-1)(\lambda+\mu)}{\lambda \mu}}}{\lambda}+\frac{\left(\vartheta-t_{0}\right)^{\frac{(\mu-1)(\lambda+\mu)}{\lambda \mu}}}{\mu}\right) .
$$


Substituting (23) into (21) yields

$$
\begin{aligned}
|f(s)||g(\vartheta)| \leq & \frac{\lambda \mu}{\lambda+\mu}\left(\frac{\left(s-t_{0}\right)^{\frac{(\lambda-1)(\lambda+\mu)}{\lambda \mu}}}{\lambda}+\frac{\left(\vartheta-t_{0}\right)^{\frac{(\mu-1)(\lambda+\mu)}{\lambda \mu}}}{\mu}\right) \\
& \times\left(\int_{t_{0}}^{s}\left|f^{\Delta}(\tau)\right|^{\lambda} \Delta \tau\right)^{\frac{1}{\lambda}}\left(\int_{t_{0}}^{\vartheta}\left|g^{\Delta}(\xi)\right|^{\mu} \Delta \xi\right)^{\frac{1}{\mu}} .
\end{aligned}
$$

Dividing both sides of (24) by the last factor $\mu\left(s-t_{0}\right)^{[(\lambda-1)(\lambda+\mu)] / \lambda \mu}+\lambda\left(\vartheta-t_{0}\right)^{[(\mu-1)(\lambda+\mu)] / \lambda \mu}$, we obtain

$$
\begin{gathered}
\frac{|f(s)||g(\vartheta)|}{\mu\left(s-t_{0}\right)^{\frac{(\lambda-1)(\lambda+\mu)}{\lambda \mu}}+\lambda\left(\vartheta-t_{0}\right)^{\frac{(\mu-1)(\lambda+\mu)}{\lambda \mu}}} \\
\leq \frac{\lambda \mu}{\lambda+\mu}\left(\int_{t_{0}}^{s}\left|f^{\Delta}(\tau)\right|^{\lambda} \Delta \tau\right)^{\frac{1}{\lambda}}\left(\int_{t_{0}}^{\vartheta}\left|g^{\Delta}(\xi)\right|^{\mu} \Delta \xi\right)^{\frac{1}{\mu}} .
\end{gathered}
$$

Integrating both sides of (25) and using (9), we find that

$$
\begin{aligned}
& \int_{t_{0}}^{x} \int_{t_{0}}^{y} \frac{|f(s)||g(\vartheta)|}{\mu\left(s-t_{0}\right)^{\frac{(\lambda-1)(\lambda+\mu)}{\lambda \mu}}+\lambda\left(\vartheta-t_{0}\right)^{\frac{(\mu-1)(\lambda+\mu)}{\lambda \mu}} \Delta s \Delta \vartheta} \\
\leq & \frac{\lambda \mu}{\lambda+\mu}\left(x-t_{0}\right)^{\frac{\lambda-1}{\lambda}}\left(y-t_{0}\right)^{\frac{\mu-1}{\mu}}\left(\int_{t_{0}}^{x}\left(\int_{t_{0}}^{s}\left|f^{\Delta}(\tau)\right|^{\lambda} \Delta \tau\right)^{\frac{1}{\lambda}} \Delta s\right) \\
& \times\left(\int_{t_{0}}^{y}\left(\int_{t_{0}}^{\vartheta}\left|g^{\Delta}(\xi)\right|^{\mu} \Delta \xi\right)^{\frac{1}{\mu}} \Delta \vartheta\right) \\
= & E(\lambda, \mu)\left(\int_{t_{0}}^{x}\left(\int_{t_{0}}^{s}\left|f^{\Delta}(\tau)\right|^{\lambda} \Delta \tau\right)^{\frac{1}{\lambda}} \Delta s\right)\left(\int_{t_{0}}^{y}\left(\int_{t_{0}}^{\vartheta}\left|g^{\Delta}(\xi)\right|^{\mu} \Delta \xi\right)^{\frac{1}{\mu}} \Delta \vartheta\right) .
\end{aligned}
$$

Applying Fubini's theorem on (26) and by taking advantage of the fact that $\sigma(\delta) \geq \delta$, we conclude that

$$
\begin{gathered}
\int_{t_{0}}^{x} \int_{t_{0}}^{y} \frac{|f(s)||g(\vartheta)|}{\mu\left(s-t_{0}\right)^{\frac{(\lambda-1)(\lambda+\mu)}{\lambda \mu}}+\lambda\left(\vartheta-t_{0}\right)^{\frac{(\mu-1)(\lambda+\mu)}{\lambda \mu}} \Delta s \Delta \vartheta} \\
\leq E(\lambda, \mu)\left(\int_{t_{0}}^{x}(\sigma(x)-s)\left|f^{\Delta}(s)\right|^{\lambda} \Delta s\right)^{\frac{1}{\lambda}}\left(\int_{t_{0}}^{y}(\sigma(y)-\vartheta)\left|g^{\Delta}(\vartheta)\right|^{\mu} \Delta \vartheta\right)^{\frac{1}{\mu}},
\end{gathered}
$$

which is equivalent to (15).

Remark 1. By setting $1 / \lambda+1 / \mu=1$ in (22), we obtain

$$
\left(s_{1}^{\omega_{1}} s_{2}^{\omega_{2}}\right) \leq \frac{1}{\omega_{1}+\omega_{2}}\left(\omega_{1} s_{1}^{\omega_{1}+\omega_{2}}+\omega_{2} s_{2}^{\omega_{1}+\omega_{2}}\right)
$$


Therefore, by applying (27) on the right-hand side of (15) in Theorem 1, we get

$$
\begin{aligned}
& \int_{t_{0}}^{x} \int_{t_{0}}^{y} \frac{|f(s)||g(\vartheta)|}{\mu\left(s-t_{0}\right) \frac{(\lambda-1)(\lambda+\mu)}{\lambda \mu}+\lambda\left(\vartheta-t_{0}\right) \frac{(\mu-1)(\lambda+\mu)}{\lambda \mu}} \Delta s \Delta \vartheta \\
\leq & \frac{\lambda \mu}{(\lambda+\mu)^{2}}\left(x-t_{0}\right)^{\frac{\lambda-1}{\lambda}}\left(y-t_{0}\right)^{\frac{\mu-1}{\mu}}\left\{\frac{1}{\lambda}\left(\int_{t_{0}}^{x}(\sigma(x)-s)\left|f^{\Delta}(s)\right|^{\lambda} \Delta s\right)^{\frac{\lambda+\mu}{\lambda \mu}}\right. \\
& \left.+\frac{1}{\mu}\left(\int_{t_{0}}^{y}(\sigma(y)-\vartheta)\left|g^{\Delta}(\vartheta)\right|^{\mu} \Delta \vartheta\right)^{\frac{\lambda+\mu}{\lambda \mu}}\right\} .
\end{aligned}
$$

Remark 2. Clearly, for $\mathbb{T}=\mathbb{Z}$ and $t_{0}=0$, inequality (28) in Remark 1 reduces to

$$
\begin{aligned}
& \sum_{s=1}^{p} \sum_{\vartheta=1}^{q} \frac{\left|a_{s}\right|\left|b_{\vartheta}\right|}{\mu s^{\frac{(\lambda-1)(\lambda+\mu)}{\lambda \mu}}+\lambda \vartheta^{\frac{(\mu-1)(\lambda+\mu)}{\lambda \mu}}} \\
& \leq \frac{\lambda \mu}{(\lambda+\mu)^{2}}(p)^{\frac{\lambda-1}{\lambda}}(q)^{\frac{\mu-1}{\mu}}\left\{\frac{1}{\lambda}\left(\sum_{s=1}^{p}(p-s+1)\left|\Delta a_{s}\right|^{\lambda}\right)^{\frac{\lambda+\mu}{\lambda \mu}}\right. \\
& \left.+\frac{1}{\mu}\left(\sum_{\vartheta=1}^{q}(q-\vartheta+1)\left|\Delta b_{\vartheta}\right|^{\mu}\right)^{\frac{\lambda+\mu}{\lambda \mu}}\right\} \text {. }
\end{aligned}
$$

where $\Delta a_{s}=a_{s+1}-a_{s}, \Delta b_{\vartheta}=b_{\vartheta+1}-b_{\vartheta}$. It is merely a similar variant of the consequence disparity in [15] Remark 1, attributed to Young and Byung.

Remark 3. For $\mathbb{T}=\mathbb{R}$ and $t_{0}=0$, Remark 1 coincides with Remark 2 in [15].

Remark 4. Inequality (15) is nothing more than a close version of the following inequality established in [28] Theorem 6,

$$
\begin{aligned}
& \int_{t_{0}}^{x} \int_{t_{0}}^{y} \frac{F(s) G(\vartheta)}{\mu\left(s-t_{0}\right)^{\frac{(\lambda-1)(\lambda+\mu)}{\lambda \mu}}+\lambda\left(\vartheta-t_{0}\right)^{\frac{(\mu-1)(\lambda+\mu)}{\lambda \mu}}} \Delta s \Delta \vartheta \\
\leq & E^{*}(\lambda, \mu)\left(\int_{t_{0}}^{x}(\sigma(x)-s)[f(s)]^{\lambda} \Delta s\right)^{\frac{1}{\lambda}} \\
& \times\left(\int_{t_{0}}^{y}(\sigma(y)-\vartheta)[g(\vartheta)]^{\mu} \Delta \vartheta\right)^{\frac{1}{\mu}}
\end{aligned}
$$

where $F(s)=\int_{t_{0}}^{s} f(\xi) \Delta \xi, G(\vartheta)=\int_{t_{0}}^{t} g(\xi) \Delta \xi$ and

$$
E^{*}(\lambda, \mu)=\frac{1}{\lambda+\mu}\left(s-t_{0}\right)^{\frac{\lambda-1}{\lambda}}\left(\vartheta-t_{0}\right)^{\frac{\mu-1}{\mu}} .
$$


Remark 5. Letting $1 / \lambda+1 / \mu=1$ in (15), then we get

$$
\begin{aligned}
& \int_{t_{0}}^{x} \int_{t_{0}}^{y} \frac{|f(s)||g(\vartheta)|}{\mu\left(s-t_{0}\right)^{\lambda-1}+\lambda\left(\vartheta-t_{0}\right)^{\mu-1}} \Delta s \Delta \vartheta \\
\leq & E^{* *}(\lambda, \mu)\left(\int_{t_{0}}^{x}(\sigma(x)-s)\left|f^{\Delta}(s)\right|^{\lambda} \Delta s\right)^{\frac{1}{\lambda}} \\
& \times\left(\int_{t_{0}}^{y}(\sigma(y)-\vartheta)\left|g^{\Delta}(\vartheta)\right|^{\mu} \Delta \vartheta\right)^{\frac{1}{\mu}},
\end{aligned}
$$

where

$$
E^{* *}(\lambda, \mu)=\frac{1}{\lambda \mu}\left(x-t_{0}\right)^{\frac{\lambda-1}{\lambda}}\left(y-t_{0}\right)^{\frac{\mu-1}{\mu}} .
$$

It is merely a similar variant of the consequence disparity in [29] Corollary 3.3, attributed to Saker et al.

Remark 6. Inequality (30) is exactly the time scale form of inequalities (1) and (3) in Theorems (1) and (2), respectively, due to B. G. Pachpatte [14].

Remark 7. As a particular state of Theorem 1 if $\mathbb{T}=\mathbb{Z}$ and $t_{0}=0$, then we have relations (8) and inequality (15) reduce to

$$
\begin{gathered}
\sum_{s=1}^{p} \sum_{\vartheta=1}^{q} \frac{\left|a_{s}\right|\left|b_{\vartheta}\right|}{\mu s} \frac{\frac{(\lambda-1)(\lambda+\mu)}{\lambda \mu}+\lambda \vartheta}{\leq E_{0}(\lambda, \mu)\left(\sum_{s=1}^{p}(p-s+1)\left|\Delta a_{s}\right|^{\lambda}\right)^{\frac{1}{\lambda}}} \\
\times\left(\sum_{\vartheta=1}^{q}(q-\vartheta+1)\left|\Delta b_{\vartheta}\right|^{\mu}\right)^{\frac{1}{\mu}},
\end{gathered}
$$

where $\Delta a_{s}=a_{s+1}-a_{s}, \Delta b_{\vartheta}=b_{\vartheta+1}-b_{\vartheta}$ and

$$
E_{0}(\lambda, \mu)=\frac{1}{\lambda+\mu}(p)^{\frac{\lambda-1}{\lambda}}(q)^{\frac{\mu-1}{\mu}}
$$

This is just a similar version of (5) that premised in the Introduction.

Remark 8. As a given state of Theorem 1 if $\mathbb{T}=\mathbb{R}$ and $t_{0}=0$, inequality (15) reduce to (6).

In what follows, we give a further generalization of (15) obtained in Theorem 1. Until giving our results, we assume that there are two functions $\Phi$ and $\Psi$ which are real-valued, nonnegative, convex, and submultiplicetive functions defined on $[0, \infty)$. A function $\chi$ is a submultiplicetive if $\chi(s \vartheta) \leq \chi(s) \chi(\vartheta)$ for $s, \vartheta \geq 0$.

Theorem 2. Let $s, \vartheta$ and $t_{0} \in \mathbb{T}, f(s) \in C_{r d}^{1}\left(\left[t_{0}, x\right)_{\mathbb{T}}, \mathbb{R}^{+}\right), g(\vartheta) \in C_{r d}^{1}\left(\left[t_{0}, y\right)_{\mathbb{T}}, \mathbb{R}^{+}\right)$and $f\left(t_{0}\right)=g\left(t_{0}\right)=0$. Suppose $h(\tau)>0$ on $\left[t_{0}, x\right)_{\mathbb{T}}$ and $l(\xi)>0$ on $\left[t_{0}, y\right)_{\mathbb{T}}$ and assume that

$$
H(s)=\int_{t_{0}}^{s}|h(\tau)| \Delta \tau \text { and } L(\vartheta)=\int_{t_{0}}^{\vartheta}|l(\xi)| \Delta \xi .
$$


Then, for $s \in\left[t_{0}, x\right)_{\mathbb{T}}$ and $\vartheta \in\left[t_{0}, y\right)_{\mathbb{T}}$, we get

$$
\begin{gathered}
\int_{t_{0}}^{x} \int_{t_{0}}^{y} \frac{\Phi(|f(s)|) \Psi(|g(\vartheta)|)}{\mu\left(s-t_{0}\right)^{\frac{(\lambda-1)(\lambda+\mu)}{\lambda \mu}}+\lambda\left(\vartheta-t_{0}\right)^{\frac{(\mu-1)(\lambda+\mu)}{\lambda \mu}} \Delta s \Delta \vartheta} \\
\leq G(\lambda, \mu)\left(\int_{t_{0}}^{x}(\sigma(x)-s)\left(|h(s)| \Phi\left(\left|\frac{f^{\Delta}(s)}{h(s)}\right|\right)\right)^{\lambda} \Delta s\right)^{\frac{1}{\lambda}} \\
\times\left(\int_{t_{0}}^{y}(\sigma(y)-\vartheta)\left(|l(\vartheta)| \Psi\left(\left|\frac{g^{\Delta}(\vartheta)}{l(\vartheta)}\right|\right)\right)^{\mu} \Delta \vartheta\right)^{\frac{1}{\mu}},
\end{gathered}
$$

where

$$
G(\lambda, \mu)=\frac{1}{\lambda+\mu}\left(\int_{t_{0}}^{x}\left(\frac{\Phi(H(s))}{H(s)}\right)^{\frac{\lambda}{\lambda-1}} \Delta s\right)^{\frac{\lambda-1}{\lambda}}\left(\int_{t_{0}}^{y}\left(\frac{\Psi(L(\vartheta))}{L(\vartheta)}\right)^{\frac{\mu}{\mu-1}} \Delta \vartheta\right)^{\frac{\mu-1}{\mu}},
$$

for $x, y \in I_{0}=\left[t_{0}, \infty\right) \cap \mathbb{T}$.

Proof. Using the two identities (17) and (18) in the proof of Theorem 1 and the properties of $\Phi$ and utilize (11), we obtain

$$
\begin{aligned}
\Phi(|f(s)|) & =\Phi\left(\frac{H(s) \int_{t_{0}}^{s}|h(\tau)|\left|\frac{f^{\Delta}(\tau)}{h(\tau)}\right| \Delta \tau}{\int_{t_{0}}^{s}|h(\tau)| \Delta \tau}\right) \\
& \leq \Phi(H(s)) \Phi\left(\frac{\int_{t_{0}}^{s}|h(\tau)| \frac{f^{\Delta}(\tau)}{h(\tau)} \mid \Delta \tau}{\int_{t_{0}}^{s}|h(\tau)| \Delta \tau}\right) \\
& \leq \frac{\Phi(H(s))}{H(s)} \int_{t_{0}}^{s}|h(\tau)| \Phi\left(\left|\frac{f^{\Delta}(\tau)}{h(\tau)}\right|\right) \Delta \tau .
\end{aligned}
$$

Further, by Hölder's integral inequality (9), we see that

$$
\begin{aligned}
\Phi(|f(s)|) & \leq \frac{\Phi(H(s))}{H(s)}\left(\int_{t_{0}}^{s}(1)^{\frac{\lambda}{\lambda-1}} \Delta \tau\right)^{\frac{\lambda-1}{\lambda}}\left(\int_{t_{0}}^{s}\left(|h(\tau)| \Phi\left(\left|\frac{f^{\Delta}(\tau)}{h(\tau)}\right|\right)\right)^{\lambda} \Delta \tau\right)^{\frac{1}{\lambda}} \\
& \leq \frac{\Phi(H(s))}{H(s)}\left(s-t_{0}\right)^{\frac{\lambda-1}{\lambda}}\left(\int_{t_{0}}^{s}\left(|h(\tau)| \Phi\left(\left|\frac{f^{\Delta}(\tau)}{h(\tau)}\right|\right)\right)^{\lambda} \Delta \tau\right)^{\frac{1}{\lambda}} .
\end{aligned}
$$

Likewise, we get

$$
\Psi(|g(\vartheta)|) \leq \frac{\Psi(L(\vartheta))}{L(\vartheta)}\left(\vartheta-t_{0}\right)^{\frac{\mu-1}{\mu}}\left(\int_{t_{0}}^{t}\left(|l(\xi)| \Psi\left(\left|\frac{g^{\Delta}(\xi)}{l(\xi)}\right|\right)\right)^{\mu} \Delta \xi\right)^{\frac{1}{\mu}} .
$$

By multiplying (36) and (37), we get

$$
\begin{aligned}
\Phi(|f(s)|) \Psi(|g(\vartheta)|) \leq & \left(s-t_{0}\right)^{\frac{\lambda-1}{\lambda}}\left(\vartheta-t_{0}\right)^{\frac{\mu-1}{\mu}} \\
& \times \frac{\Phi(H(s))}{H(s)}\left(\int_{t_{0}}^{s}\left(|h(\tau)| \Phi\left(\left|\frac{f^{\Delta}(\tau)}{h(\tau)}\right|\right)\right)^{\lambda} \Delta \tau\right)^{\frac{1}{\lambda}} \\
& \times \frac{\Psi(L(\vartheta))}{L(\vartheta)}\left(\int_{t_{0}}^{\vartheta}\left(|l(\xi)| \Psi\left(\left|\frac{g^{\Delta}(\xi)}{l(\xi)}\right|\right)\right)^{\mu} \Delta \xi\right)^{\frac{1}{\mu}} .
\end{aligned}
$$


Applying (22) on the term $\left(s-t_{0}\right)^{(\lambda-1) / \lambda} \times\left(\vartheta-t_{0}\right)^{(\mu-1) / \mu}$, gives

$$
\begin{aligned}
\Phi(|f(s)|) \Psi(|g(\vartheta)|) \leq & \frac{\lambda \mu}{\lambda+\mu}\left(\frac{\left(s-t_{0}\right)^{\frac{(\lambda-1)(\lambda+\mu)}{\lambda \mu}}}{\lambda}+\frac{\left(\vartheta-t_{0}\right)^{\frac{(\mu-1)(\lambda+\mu)}{\lambda \mu}}}{\mu}\right) \\
& \times \frac{\Phi(H(s))}{H(s)}\left(\int_{t_{0}}^{s}\left(|h(\tau)| \Phi\left(\left|\frac{f^{\Delta}(\tau)}{h(\tau)}\right|\right)\right)^{\lambda} \Delta \tau\right)^{\frac{1}{\lambda}} \\
& \times \frac{\Psi(L(\vartheta))}{L(\vartheta)}\left(\int_{t_{0}}^{\vartheta}\left(|l(\xi)| \Psi\left(\left|\frac{g^{\Delta}(\xi)}{l(\xi)}\right|\right)\right)^{\mu} \Delta \xi\right)^{\frac{1}{\mu}} .
\end{aligned}
$$

From (39), we observe that

$$
\begin{aligned}
& \frac{\Phi(|f(s)|) \Psi(|g(\vartheta)|)}{\mu\left(s-t_{0}\right)^{\frac{(\lambda-1)(\lambda+\mu)}{\lambda \mu}}+\lambda\left(\vartheta-t_{0}\right)^{\frac{(\mu-1)(\lambda+\mu)}{\lambda \mu}}} \\
\leq & \frac{1}{\lambda+\mu}\left(\frac{\Phi(H(s))}{H(s)}\left(\int_{t_{0}}^{s}\left(|h(\tau)| \Phi\left(\left|\frac{f^{\Delta}(\tau)}{h(\tau)}\right|\right)\right)^{\lambda} \Delta \tau\right)^{\frac{1}{\lambda}}\right) \\
& \times\left(\frac{\Psi(L(\vartheta))}{L(\vartheta)}\left(\int_{t_{0}}^{t}\left(|l(\xi)| \Psi\left(\left|\frac{g^{\Delta}(\xi)}{l(\xi)}\right|\right)\right)^{\mu} \Delta \xi\right)^{\frac{1}{\mu}}\right) .
\end{aligned}
$$

Integrating both sides of (40) and using (9), we find that

$$
\begin{aligned}
& \int_{t_{0}}^{x} \int_{t_{0}}^{y} \frac{\Phi(|f(s)|) \Psi(|g(\vartheta)|)}{\mu\left(s-t_{0}\right)^{\frac{(\lambda-1)(\lambda+\mu)}{\lambda \mu}}+\lambda\left(\vartheta-t_{0}\right)^{\frac{(\mu-1)(\lambda+\mu)}{\lambda \mu}} \Delta s \Delta \vartheta} \\
\leq & \frac{1}{\lambda+\mu}\left(\int_{t_{0}}^{x}\left(\frac{\Phi(H(s))}{H(s)}\right)^{\frac{\lambda}{\lambda-1}} \Delta s\right)^{\frac{\lambda-1}{\lambda}}\left(\int_{t_{0}}^{x} \int_{t_{0}}^{s}\left(|h(\tau)| \Phi\left(\left|\frac{f^{\Delta}(\tau)}{h(\tau)}\right|\right)\right)^{\lambda} \Delta \tau \Delta s\right)^{\frac{1}{\lambda}} \\
& \times\left(\int_{t_{0}}^{y}\left(\frac{\Psi(L(\vartheta))}{L(\vartheta)}\right)^{\frac{\mu}{\mu-1}} \Delta \vartheta\right)^{\frac{\mu-1}{\mu}}\left(\int_{t_{0}}^{y} \int_{t_{0}}^{\vartheta}\left(|l(\xi)| \Psi\left(\left|\frac{g^{\Delta}(\xi)}{l(\xi)}\right|\right)\right)^{\mu} \Delta \xi \Delta \vartheta\right)^{\frac{1}{\mu}}
\end{aligned}
$$


Applying Fubini's theorem on (41) and using $\sigma(\delta) \geq \delta$, we obtain

$$
\begin{aligned}
& \int_{t_{0}}^{x} \int_{t_{0}}^{y} \frac{\Phi(|f(s)|) \Psi(|g(\vartheta)|)}{\mu\left(s-t_{0}\right)^{\frac{(\lambda-1)(\lambda+\mu)}{\lambda \mu}}+\lambda\left(\vartheta-t_{0}\right)^{\frac{(\mu-1)(\lambda+\mu)}{\lambda \mu}} \Delta s \Delta \vartheta} \\
\leq & \frac{1}{\lambda+\mu}\left(\int_{t_{0}}^{x}\left(\frac{\Phi(H(s))}{H(s)}\right)^{\frac{\lambda}{\lambda-1}} \Delta s\right)^{\frac{\lambda-1}{\lambda}}\left(\int_{t_{0}}^{y}\left(\frac{\Psi(L(\vartheta))}{L(\vartheta)}\right)^{\frac{\mu}{\mu-1}} \Delta \vartheta\right)^{\frac{\mu-1}{\mu}} \\
& \times\left(\int_{t_{0}}^{x}(\sigma(x)-s)\left(|h(s)| \Phi\left(\left|\frac{f^{\Delta}(s)}{h(s)}\right|\right)\right)^{\lambda} \Delta s\right)^{\frac{1}{\lambda}} \\
& \times\left(\int_{t_{0}}^{y}(\sigma(y)-\vartheta)\left(|l(\vartheta)| \Psi\left(\left|\frac{g^{\Delta}(\vartheta)}{l(\vartheta)}\right|\right)\right)^{\mu} \Delta \vartheta\right)^{\frac{1}{\mu}} \\
= & G(\lambda, \mu)\left(\int_{t_{0}}^{x}(\sigma(x)-s)\left(|h(s)| \Phi\left(\left|\frac{f^{\Delta}(s)}{h(s)}\right|\right)\right)^{\lambda} \Delta s\right)^{\frac{1}{\lambda}} \\
& \times\left(\int_{t_{0}}^{y}(\sigma(y)-\vartheta)\left(|l(\vartheta)| \Psi\left(\left|\frac{g^{\Delta}(\vartheta)}{l(\vartheta)}\right|\right)\right)^{\mu} \Delta \vartheta\right)^{\frac{1}{\mu}} .
\end{aligned}
$$

which is equivalent to (33).

Remark 9. By applying (27) on the right-hand side of (33) in Theorem 1, then

$$
\begin{gathered}
\int_{t_{0}}^{x} \int_{t_{0}}^{y} \frac{\Phi(|f(s)|) \Psi(|g(\vartheta)|)}{\mu\left(s-t_{0}\right)^{\frac{(\lambda-1)(\lambda+\mu)}{\lambda \mu}}+\lambda\left(\vartheta-t_{0}\right)^{\frac{(\mu-1)(\lambda+\mu)}{\lambda \mu}} \Delta s \Delta \vartheta} \\
\leq G(\lambda, \mu)\left\{\frac{1}{\lambda}\left(\int_{t_{0}}^{x}(\sigma(x)-s)\left(|h(s)| \Phi\left(\left|\frac{f^{\Delta}(s)}{h(s)}\right|\right)\right)^{\lambda} \Delta s\right)^{\frac{\lambda+\mu}{\lambda \mu}}\right. \\
\left.+\frac{1}{\mu}\left(\int_{t_{0}}^{y}(\sigma(y)-\vartheta)\left(|l(\vartheta)| \Psi\left(\left|\frac{g^{\Delta}(\vartheta)}{l(\vartheta)}\right|\right)\right)^{\mu} \Delta \vartheta\right)^{\frac{\lambda+\mu}{\lambda \mu}}\right\}
\end{gathered}
$$

Remark 10. Inequality (33) is actually a related version of the following inequality in [28] Theorem 9,

$$
\begin{gathered}
\int_{t_{0}}^{x} \int_{t_{0}}^{y} \frac{\Phi(F(s)) \Psi(G(\vartheta))}{\mu\left(s-t_{0}\right)^{\frac{(\lambda-1)(\lambda+\mu)}{\lambda \mu}}+\lambda\left(\vartheta-t_{0}\right)^{\frac{(\mu-1)(\lambda+\mu)}{\lambda \mu}}} \Delta s \Delta \vartheta \\
\leq G^{*}(\lambda, \mu)\left(\int_{t_{0}}^{x}(\sigma(x)-s)\left(h(s) \Phi\left(\frac{f(s)}{h(s)}\right)\right)^{\lambda} \Delta s\right)^{\frac{1}{\lambda}} \\
\times\left(\int_{t_{0}}^{y}(\sigma(y)-\vartheta)\left(l(\vartheta) \Psi\left(\frac{g(\vartheta)}{l(\vartheta)}\right)\right)^{\mu} \Delta \vartheta\right)^{\frac{1}{\mu}}
\end{gathered}
$$

where

$$
\begin{gathered}
G^{*}(\lambda, \mu)=\frac{1}{\lambda+\mu}\left(\int_{t_{0}}^{x}\left(\frac{\Phi(H(s))}{H(s)}\right)^{\frac{\lambda}{\lambda-1}} \Delta s\right)^{\frac{\lambda-1}{\lambda}}\left(\int_{t_{0}}^{y}\left(\frac{\Psi(L(\vartheta))}{L(\vartheta)}\right)^{\frac{\mu}{\mu-1}} \Delta \vartheta\right)^{\frac{\mu-1}{\mu}}, \\
F(s)=\int_{t_{0}}^{s} f(\xi) \Delta \xi, G(\vartheta)=\int_{t_{0}}^{t} g(\xi) \Delta \xi, H(s)=\int_{t_{0}}^{s} h(\xi) \Delta \xi \text { and } L(\vartheta)=\int_{t_{0}}^{\vartheta} l(\xi) \Delta \xi .
\end{gathered}
$$


Remark 11. Letting $1 / \lambda+1 / \mu=1$ in (33), then we get

$$
\begin{aligned}
& \int_{t_{0}}^{x} \int_{t_{0}}^{y} \frac{\Phi(|f(s)|) \Psi(|g(\vartheta)|)}{\mu\left(s-t_{0}\right)^{\lambda-1}+\lambda\left(\vartheta-t_{0}\right)^{\mu-1}} \Delta s \Delta \vartheta \\
\leq & G^{* *}(\lambda, \mu)\left(\int_{t_{0}}^{x}(\sigma(x)-s)\left(|h(s)| \Phi\left(\left|\frac{f^{\Delta}(s)}{h(s)}\right|\right)\right)^{\lambda} \Delta s\right)^{\frac{1}{\lambda}} \\
& \times\left(\int_{t_{0}}^{y}(\sigma(y)-\vartheta)\left(|l(\vartheta)| \Psi\left(\left|\frac{g^{\Delta}(\vartheta)}{l(\vartheta)}\right|\right)\right)^{\mu} \Delta \vartheta\right)^{\frac{1}{\mu}},
\end{aligned}
$$

where

$$
G^{* *}(\lambda, \mu)=\frac{1}{\lambda \mu}\left(\int_{t_{0}}^{x}\left(\frac{\Phi(H(s))}{H(s)}\right)^{\frac{\lambda}{\lambda-1}} \Delta s\right)^{\frac{\lambda-1}{\lambda}}\left(\int_{t_{0}}^{y}\left(\frac{\Psi(L(\vartheta))}{L(\vartheta)}\right)^{\frac{\mu}{\mu-1}} \Delta \vartheta\right)^{\frac{\mu-1}{\mu}} .
$$

It is merely a similar variant of the consequence disparity in [29] Theorem 3.2, attributed to Saker et al.

Remark 12. As a particular state of Theorem 2 if $\mathbb{T}=\mathbb{Z}$ and $t_{0}=0$, then we have relations (8) and inequality (33) reduce to

$$
\begin{aligned}
& \sum_{s=1}^{p} \sum_{\vartheta=1}^{q} \frac{\Phi\left(\left|a_{s}\right|\right) \Psi\left(\left|b_{\vartheta}\right|\right)}{\mu s^{\frac{(\lambda-1)(\lambda+\mu)}{\lambda \mu}}+\lambda t^{\frac{(\mu-1)(\lambda+\mu)}{\lambda \mu}}} \\
\leq & G_{0}(\lambda, \mu)\left(\sum_{s=1}^{p}(p-s+1)\left(\left|a_{s}\right| \Phi\left(\left|\frac{\Delta a_{s}}{a_{s}}\right|\right)\right)^{\lambda}\right)^{\frac{1}{\lambda}} \\
& \times\left(\sum_{\vartheta=1}^{q}(q-\vartheta+1)\left(\left|b_{\vartheta}\right| \Phi\left(\left|\frac{\Delta b_{\vartheta}}{b_{\vartheta}}\right|\right)\right)^{\mu}\right)^{\frac{1}{\mu}},
\end{aligned}
$$

where $\Delta a_{s}=a_{s+1}-a_{s}, \Delta b_{\vartheta}=b_{\vartheta+1}-b_{\vartheta}$ and

$$
G_{0}(\lambda, \mu)=\frac{1}{\lambda+\mu}\left(\sum_{s=1}^{p}\left(\frac{\Phi\left(H_{s}\right)}{H_{s}}\right)^{\frac{\lambda}{\lambda-1}}\right)^{\frac{\lambda-1}{\lambda}}\left(\sum_{\vartheta=1}^{q}\left(\frac{\Psi\left(L_{\vartheta}\right)}{L_{\vartheta}}\right)^{\frac{\mu}{\mu-1}}\right)^{\frac{\mu-1}{\mu}} .
$$

For $\lambda=\mu=2$, inequality (45) is just a similar version of Pachpatte's result [13] Theorem 1.

Remark 13. As a particular case of Theorem 2 , if $\mathbb{T}=\mathbb{R}$ and $t_{0}=0$, then we have relations ( 7 ) and inequality (33) reduce to

$$
\begin{gathered}
\int_{0}^{x} \int_{0}^{y} \frac{\Phi(|f(s)|) \Psi(|g(\vartheta)|)}{\mu(s)^{\frac{(\lambda-1)(\lambda+\mu)}{\lambda \mu}}+\lambda(\vartheta)^{\frac{(\mu-1)(\lambda+\mu)}{\lambda \mu}}} d s d \vartheta \\
\leq G_{0}^{*}(\lambda, \mu)\left(\int_{0}^{x}(x-s)\left(|h(s)| \Phi\left(\left|\frac{f^{\prime}(s)}{h(s)}\right|\right)\right)^{\lambda} d s\right)^{\frac{1}{\lambda}} \\
\times\left(\int_{0}^{y}(y-\vartheta)\left(|l(\vartheta)| \Psi\left(\left|\frac{g^{\prime}(\vartheta)}{l(\vartheta)}\right|\right)\right)^{\mu} d \vartheta\right)^{\frac{1}{\mu}} .
\end{gathered}
$$

where

$$
G_{0}^{*}(\lambda, \mu)=\frac{1}{\lambda+\mu}\left(\int_{0}^{x}\left(\frac{\Phi(H(s))}{H(s)}\right)^{\frac{\lambda}{\lambda-1}} d s\right)^{\frac{\lambda-1}{\lambda}}\left(\int_{0}^{y}\left(\frac{\Psi(L(\vartheta))}{L(\vartheta)}\right)^{\frac{\mu}{\mu-1}} d \vartheta\right)^{\frac{\mu-1}{\mu}} .
$$

For $\lambda=\mu=2$, this is Pachpatte's result [13] Theorem 2. 


\subsection{The Two Dimension Version}

In the next theorems, we define the two independent variable versions of the inequalities given in Theorems 1 and 2. Throughout this paragraph, we are always assuming that $\mathbb{T}_{1}$ and $\mathbb{T}_{2}$ are two defined time scales with (i) $t_{0}, s, t, x, z \in \mathbb{T}_{1}$; (ii) $t_{0}, \vartheta, r, y, w \in \mathbb{T}_{2}$. We denote the partial delta derivatives of $u(s, \vartheta)$ with respect to $s, \vartheta$ and $s \vartheta$ by

$$
u^{\Delta_{1}}(s, \vartheta)=\frac{\partial u(s, \vartheta)}{\Delta_{1} s}, u^{\Delta_{2}}(s, \vartheta)=\frac{\partial u(s, \vartheta)}{\Delta_{2} \vartheta} \text { and } u^{\Delta_{1} \Delta_{2}}(s, \vartheta)=\frac{\partial^{2} u(s, \vartheta)}{\Delta_{2} t \Delta_{1} \vartheta}=u^{\Delta_{2} \Delta_{1}}(s, \vartheta),
$$

respectively.

Theorem 3. Let $f(s, \vartheta) \in \mathrm{CC}_{r d}^{1}\left(\left[t_{0}, x\right)_{\mathbb{T}_{1}} \times\left[t_{0}, y\right)_{\mathbb{T}_{2}}, \mathbb{R}^{+}\right), g(t, r) \in \mathrm{CC}_{r d}^{1}\left(\left[t_{0}, z\right)_{\mathbb{T}_{1}} \times\left[t_{0}, w\right)_{\mathbb{T}_{2}}, \mathbb{R}^{+}\right)$ with $f\left(s, t_{0}\right)=g\left(t, t_{0}\right)=0$ and $f\left(t_{0}, \vartheta\right)=g\left(t_{0}, r\right)=0$. Then for $(s, \vartheta) \in\left[t_{0}, x\right)_{\mathbb{T}_{1}} \times\left[t_{0}, y\right)_{\mathbb{T}_{2}}$ and $(t, r) \in\left[t_{0}, z\right)_{\mathbb{T}_{1}} \times\left[t_{0}, w\right)_{\mathbb{T}_{2}}$, one gets

$$
\begin{aligned}
& \int_{t_{0}}^{x} \int_{t_{0}}^{y}\left(\int_{t_{0}}^{z} \int_{t_{0}}^{w} \frac{|f(s, \vartheta)||g(t, r)|}{\mu\left[\left(s-t_{0}\right)\left(\vartheta-t_{0}\right)\right] \frac{(\lambda-1)(\lambda, \mu)}{\lambda \mu}+\lambda\left[\left(t-t_{0}\right)\left(r-t_{0}\right)\right]} \frac{\frac{(\mu-1)(\lambda+\mu)}{\lambda \mu}}{\lambda \mu} \Delta_{1} t \Delta_{2} r\right) \Delta_{1} s \Delta_{2} \vartheta \\
\leq & R(\lambda, \mu)\left(\int_{t_{0}}^{x} \int_{t_{0}}^{y}(\sigma(x)-s)(\sigma(y)-\vartheta)\left|\frac{\partial^{2} f(s, \vartheta)}{\Delta_{1} s \Delta_{2} \vartheta}\right|^{\lambda} \Delta_{1} s \Delta_{2} \vartheta\right)^{\frac{1}{\lambda}} \\
& \times\left(\int_{t_{0}}^{z} \int_{t_{0}}^{w}(\sigma(w)-t)(\sigma(z)-r)\left|\frac{\partial^{2} g(t, r)}{\Delta_{1} t \Delta_{2} r}\right|^{\mu} \Delta_{1} t \Delta_{2} r\right)^{\frac{1}{\mu}},
\end{aligned}
$$

where

$$
R(\lambda, \mu)=\frac{1}{\lambda+\mu}\left[\left(x-t_{0}\right)\left(y-t_{0}\right)\right]^{\frac{\lambda-1}{\lambda}}\left[\left(w-t_{0}\right)\left(z-t_{0}\right)\right]^{\frac{\mu-1}{\mu}},
$$

for $x, z \in I_{0}=\left[t_{0}, \infty\right) \cap \mathbb{T}_{1}$ and $y, w \in I_{0}^{*}=\left[t_{0}, \infty\right) \cap \mathbb{T}_{2}$.

Proof. From the hypotheses, we have the following two identities hold,

$$
\begin{aligned}
& |f(s, \vartheta)|=\int_{t_{0}}^{s} \int_{t_{0}}^{\vartheta}\left|\frac{\partial^{2} f(\xi, \eta)}{\Delta_{1} \xi \Delta_{2} \eta}\right| \Delta_{1} \xi \Delta_{2} \eta, \\
& |g(t, r)|=\int_{t_{0}}^{t} \int_{t_{0}}^{r}\left|\frac{\partial^{2} g(\sigma, \tau)}{\Delta_{1} \sigma \Delta_{2} \tau}\right| \Delta_{1} \sigma \Delta_{2} \tau .
\end{aligned}
$$

Further, by using Hölder's integral inequality (10), we find that

$$
|f(s, \vartheta)| \leq\left[\left(s-t_{0}\right)\left(\vartheta-t_{0}\right)\right]^{\frac{\lambda-1}{\lambda}}\left(\int_{t_{0}}^{s} \int_{t_{0}}^{\vartheta}\left|\frac{\partial^{2} f(\xi, \eta)}{\Delta_{1} \xi \Delta_{2} \eta}\right|^{\lambda} \Delta_{1} \xi \Delta_{2} \eta\right)^{\frac{1}{\lambda}}
$$

and

$$
|g(t, r)| \leq\left[\left(t-t_{0}\right)\left(r-t_{0}\right)\right]^{\frac{\mu-1}{\mu}}\left(\int_{t_{0}}^{t} \int_{t_{0}}^{r}\left|\frac{\partial^{2} g(\sigma, \tau)}{\Delta_{1} \sigma \Delta_{2} \tau}\right|^{\mu} \Delta_{1} \sigma \Delta_{2} \tau\right)^{\frac{1}{\mu}} .
$$


By multiplying (50) and (51), we get

$$
\begin{aligned}
|f(s, \vartheta)||g(t, r)| \leq & {\left[\left(s-t_{0}\right)\left(\vartheta-t_{0}\right)\right]^{\frac{\lambda-1}{\lambda}}\left[\left(t-t_{0}\right)\left(r-t_{0}\right)\right]^{\frac{\mu-1}{\mu}} } \\
& \times\left(\int_{t_{0}}^{s} \int_{t_{0}}^{\vartheta}\left|\frac{\partial^{2} f(\xi, \eta)}{\Delta_{1} \xi \Delta_{2} \eta}\right|^{\lambda} \Delta_{1} \xi \Delta_{2} \eta\right)^{\frac{1}{\lambda}} \\
& \times\left(\int_{t_{0}}^{t} \int_{t_{0}}^{r}\left|\frac{\partial^{2} g(\sigma, \tau)}{\Delta_{1} \sigma \Delta_{2} \tau}\right|^{\mu} \Delta_{1} \sigma \Delta_{2} \tau\right)^{\frac{1}{\mu}} .
\end{aligned}
$$

Applying (22) on the term $\left[\left(s-t_{0}\right)\left(\vartheta-t_{0}\right)\right]^{(\lambda-1) / \lambda}$ and the term $\left[\left(t-t_{0}\right)\left(r-t_{0}\right)\right]^{(\mu-1) / \mu}$, gives

$$
\begin{aligned}
& |f(s, \vartheta)||g(t, r)| \leq \frac{\lambda \mu}{\lambda+\mu}\left(\frac{\left[\left(s-t_{0}\right)\left(\vartheta-t_{0}\right) \frac{(\lambda-1)(\lambda+\mu)}{\lambda \mu}\right.}{\lambda}+\frac{\left[\left(t-t_{0}\right)\left(r-t_{0}\right)\right]^{\frac{(\mu-1)(\lambda+\mu)}{\lambda \mu}}}{\mu}\right) \\
& \times\left(\int_{t_{0}}^{s} \int_{t_{0}}^{\vartheta}\left|\frac{\partial^{2} f(\xi, \eta)}{\Delta_{1} \xi \Delta_{2} \eta}\right|^{\lambda} \Delta_{1} \xi \Delta_{2} \eta\right)^{\frac{1}{\lambda}} \\
& \times\left(\int_{t_{0}}^{t} \int_{t_{0}}^{r}\left|\frac{\partial^{2} g(\sigma, \tau)}{\Delta_{1} \sigma \Delta_{2} \tau}\right|^{\mu} \Delta_{1} \sigma \Delta_{2} \tau\right)^{\frac{1}{\mu}} \\
& =\frac{\lambda \mu}{\lambda+\mu}\left(\frac{\mu\left[\left(s-t_{0}\right)\left(\vartheta-t_{0}\right)\right] \frac{(\lambda-1)(\lambda+\mu)}{\lambda \mu}+\lambda\left[\left(t-t_{0}\right)\left(r-t_{0}\right)\right] \frac{(\mu-1)(\lambda+\mu)}{\lambda \mu}}{\lambda \mu}\right) \\
& \times\left(\int_{t_{0}}^{s} \int_{t_{0}}^{\vartheta}\left|\frac{\partial^{2} f(\xi, \eta)}{\Delta_{1} \xi \Delta_{2} \eta}\right|^{\lambda} \Delta_{1} \xi \Delta_{2} \eta\right)^{\frac{1}{\lambda}} \\
& \times\left(\int_{t_{0}}^{t} \int_{t_{0}}^{r}\left|\frac{\partial^{2} g(\sigma, \tau)}{\Delta_{1} \sigma \Delta_{2} \tau}\right|^{\mu} \Delta_{1} \sigma \Delta_{2} \tau\right)^{\frac{1}{\mu}} \text {. }
\end{aligned}
$$

Dividing both sides of (53) by $\mu\left[\left(s-t_{0}\right)\left(\vartheta-t_{0}\right)\right]^{[(\lambda-1)(\lambda+\mu)] / \lambda \mu}+\lambda\left[\left(t-t_{0}\right)\left(r-t_{0}\right)\right]^{[(\mu-1)(\lambda+\mu)] / \lambda \mu}$, we obtain

$$
\begin{aligned}
& \frac{|f(s, \vartheta)||g(t, r)|}{\mu\left[\left(s-t_{0}\right)\left(\vartheta-t_{0}\right)\right]^{\frac{(\lambda-1)(\lambda+\mu)}{\lambda \mu}}+\lambda\left[\left(t-t_{0}\right)\left(r-t_{0}\right)\right]^{\frac{(\mu-1)(\lambda+\mu)}{\lambda \mu}}} \\
& \leq \frac{1}{\lambda+\mu}\left(\int_{t_{0}}^{s} \int_{t_{0}}^{t}\left|\frac{\partial^{2} f(\xi, \eta)}{\Delta_{1} \xi \Delta_{2} \eta}\right|^{\lambda} \Delta_{1} \xi \Delta_{2} \eta\right)^{\frac{1}{\lambda}} \\
& \times\left(\int_{t_{0}}^{t} \int_{t_{0}}^{r}\left|\frac{\partial^{2} g(\sigma, \tau)}{\Delta_{1} \sigma \Delta_{2} \tau}\right|^{\mu} \Delta_{1} \sigma \Delta_{2} \tau\right)^{\frac{1}{\mu}} .
\end{aligned}
$$


Integrating both sides of (54) and using (10), we see that

$$
\begin{aligned}
& \int_{t_{0}}^{x} \int_{t_{0}}^{y}\left(\int_{t_{0}}^{z} \int_{t_{0}}^{w} \frac{|f(s, \vartheta)||g(t, r)|}{\mu\left[\left(s-t_{0}\right)\left(\vartheta-t_{0}\right)\right]} \frac{\frac{(\lambda-1)(\lambda+\mu)}{\lambda \mu}+\lambda\left[\left(t-t_{0}\right)\left(r-t_{0}\right)\right]}{\frac{(\mu-1)(\lambda+\mu)}{\lambda \mu}} \Delta_{1} t \Delta_{2} r\right) \Delta_{1} s \Delta_{2} \vartheta \\
\leq & \frac{1}{\lambda+\mu}\left[\left(x-t_{0}\right)\left(y-t_{0}\right)\right]^{\frac{\lambda-1}{\lambda}}\left[\left(z-t_{0}\right)\left(w-t_{0}\right)\right]^{\frac{\mu-1}{\mu}} \\
& \times\left(\int_{t_{0}}^{x} \int_{t_{0}}^{y}\left(\int_{t_{0}}^{s} \int_{t_{0}}^{\vartheta}\left|\frac{\partial^{2} f(\xi, \eta)}{\Delta_{1} \xi \Delta_{2} \eta}\right|^{\lambda} \Delta_{1} \xi \Delta_{2} \eta\right) \Delta_{1} s \Delta_{2} \vartheta\right)^{\frac{1}{\lambda}} \\
& \times\left(\int_{t_{0}}^{z} \int_{t_{0}}^{w}\left(\int_{t_{0}}^{t} \int_{t_{0}}^{r}\left|\frac{\partial^{2} g(\sigma, \tau)}{\Delta_{1} \sigma \Delta_{2} \tau}\right|^{\mu} \Delta_{1} \sigma \Delta_{2} \tau\right) \Delta_{1} t \Delta_{2} r\right)^{\frac{1}{\mu}} \\
= & R(\lambda, \mu)\left(\int_{t_{0}}^{x} \int_{t_{0}}^{y}\left(\int_{t_{0}}^{s} \int_{t_{0}}^{\vartheta}\left|\frac{\partial^{2} f(\xi, \eta)}{\Delta_{1} \xi \Delta_{2} \eta}\right|^{\lambda} \Delta_{1} \xi \Delta_{2} \eta\right) \Delta_{1} s \Delta_{2} \vartheta\right)^{\frac{1}{\lambda}} \\
& \times\left(\int_{t_{0}}^{z} \int_{t_{0}}^{w}\left(\int_{t_{0}}^{t} \int_{t_{0}}^{r}\left|\frac{\partial^{2} g(\sigma, \tau)}{\Delta_{1} \sigma \Delta_{2} \tau}\right|^{\mu} \Delta_{1} \sigma \Delta_{2} \tau\right) \Delta_{1} t \Delta_{2} r\right)^{\frac{1}{\mu}} .
\end{aligned}
$$

Applying Fubini's theorem on (55), we conclude that

$$
\begin{aligned}
& \int_{t_{0}}^{x} \int_{t_{0}}^{y}\left(\int_{t_{0}}^{z} \int_{t_{0}}^{w} \frac{|f(s, \vartheta)||g(t, r)|}{\mu\left[\left(s-t_{0}\right)\left(\vartheta-t_{0}\right)\right] \frac{(\lambda-1)(\lambda+\mu)}{\lambda \mu}+\lambda\left[\left(t-t_{0}\right)\left(r-t_{0}\right)\right]} \frac{(\mu-1)(\lambda+\mu)}{\lambda \mu} \Delta_{1} t \Delta_{2} r\right) \Delta_{1} s \Delta_{2} \vartheta \\
\leq & R(\lambda, \mu)\left(\int_{t_{0}}^{x} \int_{t_{0}}^{y}(x-s)(y-\vartheta)\left|\frac{\partial^{2} f(s, \vartheta)}{\Delta_{1} s \Delta_{2} \vartheta}\right|^{\lambda} \Delta_{1} s \Delta_{2} \vartheta\right)^{\frac{1}{\lambda}} \\
& \times\left(\int_{t_{0}}^{z} \int_{t_{0}}^{w}(z-t)(w-r)\left|\frac{\partial^{2} g(t, r)}{\Delta_{1} t \Delta_{2} r}\right|^{\mu} \Delta_{1} t \Delta_{2} r\right)^{\frac{1}{\mu}},
\end{aligned}
$$

by using the fact that $\sigma(\delta) \geq \delta$, one gets

$$
\begin{aligned}
& \int_{t_{0}}^{x} \int_{t_{0}}^{y}\left(\int_{t_{0}}^{z} \int_{t_{0}}^{w} \frac{|f(s, \vartheta)||g(t, r)|}{\mu\left[\left(s-t_{0}\right)\left(\vartheta-t_{0}\right)\right] \frac{(\lambda-1)(\lambda+\mu)}{\lambda \mu}+\lambda\left[\left(t-t_{0}\right)\left(r-t_{0}\right)\right]} \frac{\frac{(\mu-1)(\lambda+\mu)}{\lambda \mu}}{\lambda \mu} \Delta_{1} t \Delta_{2} r\right) \Delta_{1} s \Delta_{2} \vartheta \\
\leq & R(\lambda, \mu)\left(\int_{t_{0}}^{x} \int_{t_{0}}^{y}(\sigma(x)-s)(\sigma(y)-t)\left|\frac{\partial^{2} f(s, \vartheta)}{\Delta_{1} s \Delta_{2} \vartheta}\right|^{\lambda} \Delta_{1} s \Delta_{2} \vartheta\right) \\
& \times\left(\int_{t_{0}}^{z} \int_{t_{0}}^{w}(\sigma(w)-t)(\sigma(z)-r)\left|\frac{\partial^{2} g(t, r)}{\Delta_{1} t \Delta_{2} r}\right|^{\mu} \Delta_{1} t \Delta_{2} r\right)^{\frac{1}{\mu}},
\end{aligned}
$$

which proves (47). This completes the proof. 
Remark 14. Applying (27) on the right-hand side of (47) in Theorem 3 gives

$$
\begin{aligned}
& \int_{t_{0}}^{x} \int_{t_{0}}^{y}\left(\int_{t_{0}}^{z} \int_{t_{0}}^{w} \frac{|f(s, \vartheta)||g(t, r)|}{\mu\left[\left(s-t_{0}\right)\left(\vartheta-t_{0}\right)\right] \frac{(\lambda-1)(\lambda+\mu)}{\lambda \mu}+\lambda\left[\left(t-t_{0}\right)\left(r-t_{0}\right)\right]} \frac{\frac{(\mu-1)(\lambda+\mu)}{\lambda \mu}}{\lambda \mu} \Delta_{1} t \Delta_{2} r\right) \Delta_{1} s \Delta_{2} \vartheta \\
\leq & R(\lambda, \mu)\left\{\frac{1}{\lambda}\left(\int_{t_{0}}^{x} \int_{t_{0}}^{y}(\sigma(x)-s)(\sigma(y)-\vartheta)\left|\frac{\partial^{2} f(s, \vartheta)}{\Delta_{1} s \Delta_{2} \vartheta}\right|^{\lambda} \Delta_{1} s \Delta_{2} \vartheta\right)^{\frac{\lambda+\mu}{\lambda \mu}}\right. \\
& \left.+\frac{1}{\mu}\left(\int_{t_{0}}^{z} \int_{t_{0}}^{w}(\sigma(z)-t)(\sigma(w)-r)\left|\frac{\partial^{2} g(t, r)}{\Delta_{1} t_{2} r}\right|^{\mu} \Delta_{1} t \Delta_{2} r\right)^{\frac{\lambda+\mu}{\lambda \mu}}\right\} .
\end{aligned}
$$

Remark 15. Letting $1 / \lambda+1 / \mu=1$ in (47), then we get

$$
\begin{aligned}
& \int_{t_{0}}^{x} \int_{t_{0}}^{y}\left(\int_{t_{0}}^{z} \int_{t_{0}}^{w} \frac{|f(s, \vartheta)||g(t, r)|}{\mu\left[\left(s-t_{0}\right)\left(\vartheta-t_{0}\right)\right]^{(\lambda-1)}+\lambda\left[\left(t-t_{0}\right)\left(r-t_{0}\right)\right]^{(\mu-1)}} \Delta_{1} t \Delta_{2} r\right) \Delta_{1} s \Delta_{2} \vartheta \\
\leq & R^{*}(\lambda, \mu)\left(\int_{t_{0}}^{x} \int_{t_{0}}^{y}(\sigma(x)-s)(\sigma(y)-\vartheta)\left|\frac{\partial^{2} f(s, \vartheta)}{\Delta_{1} s \Delta_{2} \vartheta}\right|^{\lambda} \Delta_{1} s \Delta_{2} \vartheta\right)^{\frac{1}{\lambda}} \\
& \times\left(\int_{t_{0}}^{z} \int_{t_{0}}^{w}(\sigma(w)-t)(\sigma(z)-r)\left|\frac{\partial^{2} g(t, r)}{\Delta_{1} t \Delta_{2} r}\right|^{\mu} \Delta_{1} t \Delta_{2} r\right)^{\frac{1}{\mu}},
\end{aligned}
$$

where

$$
R^{*}(\lambda, \mu)=\frac{1}{\lambda \mu}\left[\left(x-t_{0}\right)\left(y-t_{0}\right)\right]^{\frac{\lambda-1}{\lambda}}\left[\left(w-t_{0}\right)\left(z-t_{0}\right)\right]^{\frac{\mu-1}{\mu}},
$$

which is exactly the time scale version of inequalities (8) and (10) in Theorems (3) and (4), respectively, due to B. G. Pachpatte [14].

Remark 16. As a particular state of Theorem 3 if $\mathbb{T}_{1}=\mathbb{T}_{2}=\mathbb{Z}$ and $t_{0}=0$, then we have relations (8) and inequality (47) reduce to

$$
\begin{aligned}
& \sum_{s=1}^{x} \sum_{\vartheta=1}^{y}\left(\sum_{t=1}^{z} \sum_{r=1}^{w} \frac{\left|a_{s, \vartheta}\right|\left|b_{t, r}\right|}{\mu(s \vartheta)^{\frac{(\lambda-1)(\lambda+\mu)}{\lambda \mu}}+\lambda(t r)^{\frac{(\lambda-1)(\lambda+\mu)}{\lambda \mu}}}\right) \\
\leq & R_{0}(\lambda, \mu)\left(\sum_{s=1}^{x} \sum_{\vartheta=1}^{y}(x-s+1)(y-\vartheta+1)\left|\Delta_{2} \Delta_{1}\left(a_{s, \vartheta}\right)\right|^{\lambda}\right)^{\frac{1}{\lambda}} \\
& \times\left(\sum_{t=1}^{z} \sum_{r=1}^{w}(z-t+1)(w-r+1)\left|\Delta_{2} \Delta_{1}\left(b_{t, r}\right)\right|^{\mu}\right)^{\frac{1}{\mu}},
\end{aligned}
$$

where the operators $\Delta_{1}\left(a_{s, \vartheta}\right)=a_{s+1, \vartheta}-a_{s, \vartheta}, \Delta_{1}\left(b_{t, r}\right)=b_{t+1, r}-b_{t, r}, \Delta_{2} \Delta_{1}\left(a_{s, \vartheta}\right)=\Delta_{2}\left(\Delta_{1}\left(a_{s, \vartheta}\right)\right)=$ $\Delta_{1}\left(\Delta_{2}\left(a_{s, \vartheta}\right)\right), \Delta_{2} \Delta_{1}\left(b_{t, r}\right)=\Delta_{2}\left(\Delta_{1}\left(b_{t, r}\right)\right)=\Delta_{1}\left(\Delta_{2}\left(b_{t, r}\right)\right)$ and

$$
R_{0}(\lambda, \mu)=\frac{1}{\lambda+\mu}(x y)^{\frac{\lambda-1}{\lambda}}(z w)^{\frac{\mu-1}{\mu}} .
$$

It is merely a similar variant of the consequence disparity in [15] Theorem 2.3. 
Remark 17. As a particular state of Theorem 3 , if $\mathbb{T}_{1}=\mathbb{T}_{2}=\mathbb{R}$ and $t_{0}=0$, we have relations (7) and inequality (47) reduce to

$$
\begin{aligned}
& \int_{0}^{x} \int_{0}^{y}\left(\int_{0}^{z} \int_{0}^{w} \frac{|f(s, \vartheta)||g(t, r)|}{\mu(s \vartheta)^{\frac{(\lambda-1)(\lambda+\mu)}{\lambda \mu}}+\lambda(t r)^{\frac{(\lambda-1)(\lambda+\mu)}{\lambda \mu}}} d t d r\right) d s d \vartheta \\
\leq & R_{0}^{*}(\lambda, \mu)\left(\int_{0}^{x} \int_{0}^{y}(x-s)(y-\vartheta)\left|D_{2} D_{1} f(s, \vartheta)\right|^{\lambda} d s d \vartheta\right)^{\frac{1}{\lambda}} \\
& \times\left(\int_{0}^{z} \int_{0}^{w}(z-t)(w-r)\left|D_{2} D_{1} g(t, r)\right|^{\mu} d t d r\right)^{\frac{1}{\mu}},
\end{aligned}
$$

where

$$
R_{0}^{*}(\lambda, \mu)=\frac{1}{\lambda+\mu}(x y)^{\frac{\lambda-1}{\lambda}}(z w)^{\frac{\mu-1}{\mu}}
$$

and $D_{1} u(s, \vartheta)=(\partial / \partial s) u(s, \vartheta), D_{2} u(s, \vartheta)=(\partial / \partial \vartheta) u(s, \vartheta), D_{2} D_{1} u(s, \vartheta)=D_{2} D_{1} u(s, \vartheta)=$ $\left(\partial^{2} / \partial s \partial \vartheta\right) u(s, \vartheta)$, which is the same result inequality due to Young and Byung in [15] Theorem 2.4.

Theorem 4. Let $f(s, \vartheta)$ and $g(t, r)$ with $f\left(s, t_{0}\right)=g\left(t, t_{0}\right)=0$ and $f\left(t_{0}, \vartheta\right)=g\left(t_{0}, r\right)=0$, be as in Theorem 3 and $h(\xi, \eta)>0, l(\sigma, \tau)>0$. Furthermore, assume that

$$
H(s, \vartheta)=\int_{t_{0}}^{s} \int_{t_{0}}^{\vartheta}|h(\xi, \eta)| \Delta_{1} \xi \Delta_{2} \eta \quad \text { and } L(t, r)=\int_{t_{0}}^{t} \int_{t_{0}}^{r}|l(\sigma, \tau)| \Delta_{1} \sigma \Delta_{2} \tau \text {. }
$$

Then for $(s, v) \in\left[t_{0}, x\right)_{\mathbb{T}_{1}} \times\left[t_{0}, y\right)_{\mathbb{T}_{2}}$ and $(t, r) \in\left[t_{0}, z\right)_{\mathbb{T}_{1}} \times\left[t_{0}, w\right)_{\mathbb{T}_{2}}$, one gets

$$
\begin{aligned}
& \int_{t_{0}}^{x} \int_{t_{0}}^{y}\left(\int_{t_{0}}^{z} \int_{t_{0}}^{w} \frac{\Phi(|f(s, \vartheta)|) \Psi(|g(t, r)|)}{\mu\left[\left(s-t_{0}\right)\left(\vartheta-t_{0}\right)\right] \frac{(\lambda-1)(\lambda+\mu)}{\lambda \mu}+\lambda\left[\left(t-t_{0}\right)\left(r-t_{0}\right)\right]} \frac{\frac{(\mu-1)(\lambda+\mu)}{\lambda \mu}}{\lambda \mu} \Delta_{1} t \Delta_{2} r\right) \Delta_{1} s \Delta_{2} \vartheta \\
\leq & S(\lambda, \mu)\left(\int_{t_{0}}^{x} \int_{t_{0}}^{y}(\sigma(x)-s)(\sigma(y)-\vartheta)\left(|h(s, \vartheta)| \Phi\left(\left|\frac{\frac{\partial^{2} f(s, \vartheta)}{\Delta_{1} s \Delta_{2} \vartheta}}{h(s, \vartheta)}\right|\right)\right)^{\lambda} \Delta_{1} s \Delta_{2} \vartheta\right)^{\frac{1}{\lambda}} \\
& \times\left(\int_{t_{0}}^{z} \int_{t_{0}}^{w}(\sigma(z)-t)(\sigma(w)-r)\left(|l(t, r)| \Psi\left(\left|\frac{\frac{\partial}{2} g(t, r)_{\Delta_{1} t \Delta_{2} r}}{l(t, r)}\right|\right)\right)^{\mu} \Delta_{1} t \Delta_{2} r\right)^{\frac{1}{\mu}},
\end{aligned}
$$

where

$$
S(\lambda, \mu)=\frac{1}{\lambda+\mu}\left(\int_{t_{0}}^{x} \int_{t_{0}}^{y}\left(\frac{\Phi(H(s, \vartheta))}{H(s, t)}\right)^{\frac{\lambda}{\lambda-1}} \Delta_{1} s \Delta_{2} \vartheta\right)^{\frac{\lambda-1}{\lambda}}\left(\int_{t_{0}}^{z} \int_{t_{0}}^{w}\left(\frac{\Psi(L(t, r))}{L(t, r)}\right)^{\frac{\mu}{\mu-1}} \Delta_{1} t \Delta_{2} r\right)^{\frac{\mu-1}{\mu}} .
$$


Proof. Using the two identities (48) and (49) in the proof of Theorem 3 and the properties of the function $\Phi$ and utilize (12), we obtain

$$
\begin{aligned}
\Phi(|f(s, \vartheta)|) & =\Phi\left(\frac{H(s, \vartheta) \int_{t_{0}}^{s} \int_{t_{0}}^{\vartheta}|h(\xi, \eta)|\left(\left|\frac{\frac{\partial^{2} f(\xi, \eta)}{\Delta_{1} \xi \Delta_{2} \eta}}{h(\xi, \eta)}\right|\right) \Delta_{1} \xi \Delta_{2} \eta}{\int_{t_{0}}^{s} \int_{t_{0}}^{\vartheta}|h(\xi, \eta)| \Delta_{1} \xi \Delta_{2} \eta}\right) \\
& \leq \Phi(H(s, \vartheta)) \Phi\left(\frac{\int_{t_{0}}^{s} \int_{t_{0}}^{\vartheta}|h(\xi, \eta)|\left(\left|\frac{\frac{\partial^{2} f(\xi, \eta)}{\Delta_{1} \xi \Delta_{2} \eta}}{h(\xi, \eta)}\right|\right) \Delta_{1} \xi \Delta_{2} \eta}{\int_{t_{0}}^{s} \int_{t_{0}}^{\vartheta}|h(\xi, \eta)| \Delta_{1} \xi \Delta_{2} \eta}\right) \\
& \leq \frac{\Phi(H(s, \vartheta))}{H(s, \vartheta)} \int_{t_{0}}^{s} \int_{t_{0}}^{\vartheta}|h(\xi, \eta)| \Phi\left(\left|\frac{\frac{\partial^{2} f(\xi, \eta)}{\Delta_{1} \xi \Delta_{2} \eta}}{h(\xi, \eta)}\right|\right) \Delta_{1} \xi \Delta_{2} \eta .
\end{aligned}
$$

Applying (10) with indices $\lambda$ and $\lambda /(\lambda-1)$ on the right-hand side of (61), we have

$$
\begin{aligned}
\Phi(|f(s, \vartheta)|) \leq & {\left[\left(s-t_{0}\right)\left(\vartheta-t_{0}\right)\right]^{\frac{\lambda-1}{\lambda}} \frac{\Phi(H(s, \vartheta))}{H(s, \vartheta)} } \\
& \times\left(\int_{t_{0}}^{s} \int_{t_{0}}^{\vartheta}\left(|h(\xi, \eta)| \Phi\left(\left|\frac{\frac{\partial^{2} f(\xi, \eta)}{\Delta_{1} \xi \Delta_{2} \eta}}{h(\xi, \eta)}\right|\right)\right)^{\lambda} \Delta_{1} \xi \Delta_{2} \eta\right)^{\frac{1}{\lambda}} .
\end{aligned}
$$

Analogously,

$$
\begin{aligned}
\Psi(|g(t, r)|) \leq & {\left[\left(t-t_{0}\right)\left(r-t_{0}\right)\right]^{\frac{\mu-1}{\mu}} \frac{\Psi(L(t, r))}{L(t, r)} } \\
& \times\left(\int_{t_{0}}^{t} \int_{t_{0}}^{r}\left(|l(\sigma, \tau)| \Psi\left(\left|\frac{\frac{\partial^{2} g(\sigma, \tau)}{\Delta_{1} \sigma \Delta_{2} \tau}}{l(\sigma, \tau)}\right|\right)\right)^{\mu} \Delta_{1} \sigma \Delta_{2} \tau\right)^{\frac{1}{\mu}} .
\end{aligned}
$$

Thus, form (62) and (63), it can be acquired that

$$
\begin{aligned}
& \Phi(|f(s, \vartheta)|) \Psi(|g(t, r)|) \\
& \leq \quad\left[\left(s-t_{0}\right)\left(\vartheta-t_{0}\right)\right]^{\frac{\lambda-1}{\lambda}}\left[\left(t-t_{0}\right)\left(r-t_{0}\right)\right]^{\frac{\mu-1}{\mu}} \\
& \times \frac{\Phi(H(s, \vartheta))}{H(s, \vartheta)}\left(\int_{t_{0}}^{s} \int_{t_{0}}^{\vartheta}\left(|h(\xi, \eta)| \Phi\left(\left|\frac{\frac{\partial^{2} f(\xi, \eta)}{\Delta_{1} \xi \Delta_{2} \eta}}{h(\xi, \eta)}\right|\right)\right)^{\lambda} \Delta_{1} \xi \Delta_{2} \eta\right)^{\frac{1}{\lambda}} \\
& \times \\
& \times \frac{\Psi(L(t, r))}{L(t, r)}\left(\int_{t_{0}}^{t} \int_{t_{0}}^{r}\left(|l(\sigma, \tau)| \Psi\left(\left|\frac{\frac{\partial^{2} g(\sigma, \tau)}{\Delta_{1} \sigma \Delta_{2} \tau}}{l(\sigma, \tau)}\right|\right)\right)^{\mu} \Delta_{1} \sigma \Delta_{2} \tau\right)^{\frac{1}{\mu}} .
\end{aligned}
$$


Applying (22) on the term $\left[\left(s-t_{0}\right)\left(\vartheta-t_{0}\right)\right]^{(\lambda-1) / \lambda}$ and the term $\left[\left(t-t_{0}\right)\left(r-t_{0}\right)\right]^{(\mu-1) / \mu}$ gives

$$
\begin{aligned}
& \Phi(|f(s, \vartheta)|) \Psi(|g(t, r)|) \\
\leq & \frac{\lambda \mu}{\lambda+\mu}\left(\frac{\left[\left(s-t_{0}\right)\left(\vartheta-t_{0}\right)\right]^{\frac{(\lambda-1)(\lambda+\mu)}{\lambda \mu}}}{\lambda}+\frac{\left[\left(t-t_{0}\right)\left(r-t_{0}\right)\right]^{\frac{(\mu-1)(\lambda+\mu)}{\lambda \mu}}}{\mu}\right) \\
\times & \frac{\Phi(H(s, \vartheta))}{H(s, \vartheta)}\left(\int_{t_{0}}^{s} \int_{t_{0}}^{\vartheta}\left(|h(\xi, \eta)| \Phi\left(\left|\frac{\frac{\partial^{2} f(\xi, \eta)}{\Delta_{1} \xi \Delta_{2} \eta}}{h(\xi, \eta)}\right|\right)\right)^{\lambda} \Delta_{1} \xi \Delta_{2} \eta\right)^{\frac{1}{\lambda}} \\
\times & \frac{\Psi(L(t, r))}{L(t, r)}\left(\int_{t_{0}}^{t} \int_{t_{0}}^{r}\left(|l(\sigma, \tau)| \Psi\left(\left|\frac{\partial^{2} g(\sigma, \tau)}{\Delta_{1} \sigma \Delta_{2} \tau}\right|\right)\right)^{\mu} \Delta_{1} \sigma \Delta_{2} \tau\right)^{\frac{1}{\mu}} .
\end{aligned}
$$

From (65), we observe that

$$
\begin{aligned}
& \frac{\Phi(|f(s, \vartheta)|) \Psi(|g(t, r)|)}{\mu\left[\left(s-t_{0}\right)\left(\vartheta-t_{0}\right)\right]^{\frac{(\lambda-1)(\lambda+\mu)}{\lambda \mu}}+\lambda\left[\left(t-t_{0}\right)\left(r-t_{0}\right)\right]^{\frac{(\mu-1)(\lambda+\mu)}{\lambda \mu}}} \\
& \leq \frac{1}{\lambda+\mu} \frac{\Phi(H(s, \vartheta))}{H(s, \vartheta)}\left(\int_{t_{0}}^{s} \int_{t_{0}}^{\vartheta}\left(|h(\xi, \eta)| \Phi\left(\left|\frac{\frac{\partial^{2} f(\xi, \eta)}{\Delta_{1} \xi \Delta_{2} \eta}}{h(\xi, \eta)}\right|\right)\right)^{\lambda} \Delta_{1} \xi \Delta_{2} \eta\right)^{\frac{1}{\lambda}} \\
& \times \frac{\Psi(L(t, r))}{L(t, r)}\left(\int_{t_{0}}^{t} \int_{t_{0}}^{r}\left(|l(\sigma, \tau)| \Psi\left(\left|\frac{\partial^{2} g(\sigma, \tau)}{\Delta_{1} \sigma \Delta_{2} \tau}\right|\right)\right)^{\mu} \Delta_{1} \sigma \Delta_{2} \tau\right)^{\frac{1}{\mu}} .
\end{aligned}
$$

Integrating both sides of (66) and using (10) again with respect to $\lambda, \lambda /(\lambda-1)$ and $\mu, \mu /(\mu-1)$, respectively, we may write

$$
\begin{aligned}
& \int_{t_{0}}^{x} \int_{t_{0}}^{y}\left(\int_{t_{0}}^{z} \int_{t_{0}}^{w} \frac{\Phi(|f(s, \vartheta)|) \Psi(|g(t, r)|)}{\mu\left[\left(s-t_{0}\right)\left(\vartheta-t_{0}\right)\right] \frac{(\lambda-1)(\lambda+\mu)}{\lambda \mu}+\lambda\left[\left(t-t_{0}\right)\left(r-t_{0}\right)\right]} \frac{(\mu-1)(\lambda+\mu)}{\lambda \mu} \Delta_{1} t \Delta_{2} r\right) \Delta_{1} s \Delta_{2} \vartheta \\
& \leq \frac{1}{\lambda+\mu}\left(\int_{t_{0}}^{x} \int_{t_{0}}^{y}\left(\frac{\Phi(H(s, \vartheta))}{H(s, \vartheta)}\right)^{\frac{\lambda}{\lambda-1}} \Delta_{1} s \Delta_{2} \vartheta\right)^{\frac{\lambda-1}{\lambda}}\left(\int_{t_{0}}^{z} \int_{t_{0}}^{w}\left(\frac{\Psi(L(t, r))}{L(t, r)}\right)^{\frac{\mu}{\mu-1}} \Delta_{1} t \Delta_{2} r\right)^{\frac{\mu-1}{\mu}} \\
& \times\left(\int_{t_{0}}^{x} \int_{t_{0}}^{y}\left(\int_{t_{0}}^{s} \int_{t_{0}}^{\vartheta}\left(|h(\xi, \eta)| \Phi\left(\left|\frac{\frac{\partial^{2} f(\xi, \eta)}{\Delta_{1} \xi \Delta_{2} \eta}}{h(\xi, \eta)}\right|\right)\right)^{\lambda} \Delta_{1} \xi \Delta_{2} \eta\right) \Delta_{1} s \Delta_{2} \vartheta\right)^{\frac{1}{\lambda}} \\
& \times\left(\int_{t_{0}}^{z} \int_{t_{0}}^{w}\left(\int_{t_{0}}^{t} \int_{t_{0}}^{r}\left(|l(\sigma, \tau)| \Psi\left(\left|\frac{\frac{\partial^{2} g(\sigma, \tau)}{\Delta_{1} \sigma \Delta_{2} \tau}}{l(\sigma, \tau)}\right|\right)\right)^{\mu} \Delta_{1} \xi \Delta_{2} \eta\right) \Delta_{1} t \Delta_{2} r\right)^{\frac{1}{\mu}} \\
& =S(\lambda, \mu)\left(\int_{t_{0}}^{x} \int_{t_{0}}^{y}\left(\int_{t_{0}}^{s} \int_{t_{0}}^{\vartheta}\left(|h(\xi, \eta)| \Phi\left(\left|\frac{\frac{\partial^{2} f(\xi, \eta)}{\Delta_{1} \xi \Delta_{2} \eta}}{h(\xi, \eta)}\right|\right)\right)^{\lambda} \Delta_{1} \xi \Delta_{2} \eta\right) \Delta_{1} s \Delta_{2} \vartheta\right)^{\frac{1}{\lambda}} \\
& \times\left(\int_{t_{0}}^{z} \int_{t_{0}}^{w}\left(\int_{t_{0}}^{t} \int_{t_{0}}^{r}\left(|l(\sigma, \tau)| \Psi\left(\left|\frac{\frac{\partial^{2} g(\sigma, \tau)}{\Delta_{1} \sigma \Delta_{2} \tau}}{l(\sigma, \tau)}\right|\right)\right)^{\mu} \Delta_{1} \sigma \Delta_{2} \tau\right) \Delta_{1} t \Delta_{2} r\right)^{\frac{1}{\mu}} .
\end{aligned}
$$


Applying Fubini's theorem on (67) and using $\sigma(\delta) \geq \delta$, we get

$$
\begin{aligned}
& \int_{t_{0}}^{x} \int_{t_{0}}^{y}\left(\int_{t_{0}}^{z} \int_{t_{0}}^{w} \frac{\Phi(|f(s, \vartheta)|) \Psi(|g(t, r)|)}{\mu\left[\left(s-t_{0}\right)\left(\vartheta-t_{0}\right)\right] \frac{(\lambda-1)(\lambda+\mu)}{\lambda \mu}+\lambda\left[\left(t-t_{0}\right)\left(r-t_{0}\right)\right]} \frac{\frac{(\mu-1)(\lambda+\mu)}{\lambda \mu}}{\lambda \mu} \Delta_{1} t \Delta_{2} r\right) \Delta_{1} s \Delta_{2} \vartheta \\
& \leq S(\lambda, \mu)\left(\int_{t_{0}}^{x} \int_{t_{0}}^{y}(x-s)(y-\vartheta)\left(|h(s, \vartheta)| \Phi\left(\left|\frac{\frac{\partial^{2} f(s, \vartheta)}{\Delta_{1} s \Delta_{2} \vartheta}}{h(s, t)}\right|\right)\right)^{\lambda} \Delta_{1} s \Delta_{2} \vartheta\right)^{\frac{1}{\lambda}} \\
& \times\left(\int_{t_{0}}^{z} \int_{t_{0}}^{w}(z-t)(w-r)\left(\mid\left(l(t, r) \mid \Psi\left(\left|\frac{\frac{\partial^{2} g(t, r)}{\Delta_{1} t \Delta_{2} r}}{l(t, r)}\right|\right)\right)^{\mu} \Delta_{1} t \Delta_{2} r\right)^{\frac{1}{\mu}}\right. \\
& \leq S(\lambda, \mu)\left(\int_{t_{0}}^{x} \int_{t_{0}}^{y}(\sigma(x)-s)(\sigma(y)-\vartheta)\left(|h(s, \vartheta)| \Phi\left(\left|\frac{\frac{\partial^{2} f(s, \vartheta)}{\Delta_{1} s \Delta_{2} \vartheta}}{h(s, \vartheta)}\right|\right)\right)^{\lambda} \Delta_{1} s \Delta_{2} \vartheta\right)^{\frac{1}{\lambda}} \\
& \times\left(\int_{t_{0}}^{z} \int_{t_{0}}^{w}(\sigma(z)-t)(\sigma(w)-r)\left(\mid\left(l(t, r) \mid \Psi\left(\left|\frac{\frac{\partial^{2} g(t, r)}{\Delta_{1} t \Delta_{2} r}}{l(t, r)}\right|\right)\right)^{\mu} \Delta_{1} t \Delta_{2} r\right)^{\frac{1}{\mu}},\right.
\end{aligned}
$$

which is (60). This completes the proof.

Remark 18. By applying (27) on the right-hand side of (60) in Theorem 4, then

$$
\begin{aligned}
& \int_{t_{0}}^{x} \int_{t_{0}}^{y}\left(\int_{t_{0}}^{z} \int_{t_{0}}^{w} \frac{\Phi(|f(s, \vartheta)|) \Psi(|g(t, r)|)}{\mu\left[\left(s-t_{0}\right)\left(t-t_{0}\right)\right]} \frac{(\lambda-1)(\lambda+\mu)}{\lambda \mu}+\lambda\left[\left(t-t_{0}\right)\left(r-t_{0}\right)\right] \frac{(\mu-1)(\lambda+\mu)}{\lambda \mu} \Delta_{1} t \Delta_{2} r\right) \Delta_{1} s \Delta_{2} t \\
\leq & S(\lambda, \mu)\left\{\frac{1}{\lambda}\left(\int_{t_{0}}^{x} \int_{t_{0}}^{y}(\sigma(x)-s)(\sigma(y)-\vartheta)\left(|h(s, \vartheta)| \Phi\left(\left|\frac{\frac{\partial^{2} f(s, \vartheta)}{\Delta_{1} s_{2} t}}{h(s, \vartheta)}\right|\right)\right)^{\lambda} \Delta_{1} s \Delta_{2} \vartheta\right)^{\frac{\lambda+\mu}{\lambda \mu}}\right. \\
& +\frac{1}{\mu}\left(\int_{t_{0}}^{z} \int_{t_{0}}^{w}(\sigma(z)-t)(\sigma(w)-r)\left(\mid\left(l(t, r) \mid \Psi\left(\left|\frac{\frac{\partial^{2} g(t, r)}{\Delta_{1}+\Delta_{2} r}}{l(t, r)}\right|\right)\right)^{\mu} \Delta_{1} t \Delta_{2} r\right)^{\frac{\lambda+\mu}{\lambda \mu}}\right\} .
\end{aligned}
$$

Remark 19. Letting $1 / \lambda+1 / \mu=1$ in (60), then we get

$$
\begin{aligned}
& \int_{t_{0}}^{x} \int_{t_{0}}^{y}\left(\int_{t_{0}}^{z} \int_{t_{0}}^{w} \frac{\Phi(|f(s, \vartheta)|) \Psi(|g(t, r)|)}{\mu\left[\left(s-t_{0}\right)\left(\vartheta-t_{0}\right)\right]^{(\lambda-1)}+\lambda\left[\left(t-t_{0}\right)\left(r-t_{0}\right)\right]^{(\mu-1)}} \Delta_{1} t \Delta_{2} r\right) \Delta_{1} s \Delta_{2} \vartheta \\
\leq & S^{*}(\lambda, \mu)\left(\int _ { t _ { 0 } } ^ { x } \int _ { t _ { 0 } } ^ { y } ( \sigma ( x ) - s ) ( \sigma ( y ) - \vartheta ) \left(|h(s, \vartheta)| \Phi\left(\mid \frac{\left.\left.\frac{\partial^{2} f(s, \vartheta)}{\frac{\Delta_{1} s, \vartheta}{h(s, \vartheta)}} \mid\right)\right)^{\lambda} \Delta_{1} s \Delta_{2} \vartheta}{h}\right)^{\frac{1}{\lambda}}\right.\right. \\
& \times\left(\int_{t_{0}}^{z} \int_{t_{0}}^{w}(\sigma(z)-t)(\sigma(w)-r)\left(\mid\left(l(t, r) \mid \Psi\left(\left|\frac{\frac{\partial^{2} g_{1}(t, r)}{\Delta_{1} \Delta_{2} r}}{l(t, r)}\right|\right)\right)^{\mu} \Delta_{1} t \Delta_{2} r\right)^{\frac{1}{\mu}}\right.
\end{aligned}
$$

where

$$
S^{*}(\lambda, \mu)=\frac{1}{\lambda \mu}\left(\int_{t_{0}}^{x} \int_{t_{0}}^{y}\left(\frac{\Phi(H(s, \vartheta))}{H(s, \vartheta)}\right)^{\frac{\lambda}{\lambda-1}} \Delta_{1} s \Delta_{2} \vartheta\right)^{\frac{\lambda-1}{\lambda}}\left(\int_{t_{0}}^{z} \int_{t_{0}}^{w}\left(\frac{\Psi(L(t, r))}{L(t, r)}\right)^{\frac{\mu}{\mu-1}} \Delta_{1} t \Delta_{2} r\right)^{\frac{\mu-1}{\mu}} .
$$


Remark 20. As a particular state of Theorem 4 if $\mathbb{T}_{1}=\mathbb{T}_{2}=\mathbb{Z}$ and $t_{0}=0$, then we have relation (8) and inequality (60) reduce to

$$
\begin{aligned}
& \sum_{s=1}^{x} \sum_{\vartheta=1}^{y}\left(\sum_{t=1}^{z} \sum_{r=1}^{w} \frac{\Phi\left(\left|a_{s, \vartheta}\right|\right) \Psi\left(\left|b_{t, r}\right|\right)}{\mu(s \vartheta)^{\frac{(\lambda-1)(\lambda+\mu)}{\lambda \mu}}+\lambda(t r)^{\frac{(\lambda-1)(\lambda+\mu)}{\lambda \mu}}}\right) \\
\leq & S_{0}(\lambda, \mu)\left(\sum_{s=1}^{x} \sum_{\vartheta=1}^{y}(x-s+1)(y-t+1)\left(|h(s, \vartheta)| \Phi\left(\left|\frac{\Delta_{2} \Delta_{1}\left(a_{s, \vartheta}\right)}{h(s, \vartheta)}\right|\right)\right)^{\lambda}\right)^{\frac{1}{\lambda}} \\
& \times\left(\sum_{t=1}^{z} \sum_{r=1}^{w}(z-t+1)(w-r+1)\left(\mid\left(l(t, r) \mid \Psi\left(\left|\frac{\Delta_{2} \Delta_{1} b_{t, r}}{l(t, r)}\right|\right)\right)^{\mu}\right)^{\frac{1}{\mu}},\right.
\end{aligned}
$$

where the operators $\Delta_{1}\left(a_{s, \vartheta}\right)=a_{s+1, \vartheta}-a_{s, \vartheta}, \Delta_{1}\left(b_{t, r}\right)=b_{t+1, r}-b_{t, r}, \Delta_{2} \Delta_{1}\left(a_{s, \vartheta}\right)=\Delta_{2}\left(\Delta_{1}\left(a_{s, \vartheta}\right)\right)=$ $\Delta_{1}\left(\Delta_{2}\left(a_{s, \vartheta}\right)\right), \Delta_{2} \Delta_{1} b_{t, r}=\Delta_{2}\left(\Delta_{1}\left(b_{t, r}\right)\right)=\Delta_{1}\left(\Delta_{2}\left(b_{t, r}\right)\right)$ and

$$
S_{0}(\lambda, \mu)=\frac{1}{\lambda+\mu}\left(\sum_{s=1}^{x} \sum_{\vartheta=1}^{y}\left(\frac{\Phi\left(H_{s, \vartheta}\right)}{H_{s, \vartheta}}\right)^{\frac{\lambda}{\lambda-1}}\right)^{\frac{\lambda-1}{\lambda}}\left(\sum_{t=1}^{z} \sum_{r=1}^{w}\left(\frac{\Psi\left(L_{t, r}\right)}{L_{t, r}}\right)^{\frac{\mu}{\mu-1}}\right)^{\frac{\mu-1}{\mu}} .
$$

Remark 21. As a particular state of Theorem 4 if $\mathbb{T}_{1}=\mathbb{T}_{2}=\mathbb{R}$ and $t_{0}=0$, we have relations (7) and inequality (60) reduce to

$$
\begin{aligned}
& \int_{0}^{x} \int_{0}^{y}\left(\int_{0}^{z} \int_{0}^{w} \frac{\Phi(|f(s, \vartheta)|) \Psi(|g(t, r)|)}{\mu(s \vartheta)^{\frac{(\lambda-1)(\lambda+\mu)}{\lambda \mu}}+\lambda(t r) \frac{(\lambda-1)(\lambda+\mu)}{\lambda \mu}} d t d r\right) d s d \vartheta \\
\leq & S_{0}^{*}(\lambda, \mu)\left(\int_{0}^{x} \int_{0}^{y}(x-s)(y-\vartheta)\left(|h(s, \vartheta)| \Phi\left(\left|\frac{D_{2} D_{1} f(s, \vartheta)}{h(s, \vartheta)}\right|\right)\right)^{\lambda} d s d \vartheta\right)^{\frac{1}{\lambda}} \\
& \times\left(\int_{0}^{z} \int_{0}^{w}(z-t)(w-r)\left(\mid\left(l(t, r) \mid \Psi\left(\left|\frac{D_{2} D_{1} g(t, r)}{l(t, r)}\right|\right)\right)^{\mu} d t d r\right)^{\frac{1}{\mu}},\right.
\end{aligned}
$$

where

$$
S_{0}^{*}(\lambda, \mu)=\frac{1}{\lambda+\mu}\left(\int_{0}^{x} \int_{0}^{y}\left(\frac{\Phi(H(s, \vartheta))}{H(s, \vartheta)}\right)^{\frac{\lambda}{\lambda-1}} d s d \vartheta\right)^{\frac{\lambda-1}{\lambda}}\left(\int_{0}^{z} \int_{0}^{w}\left(\frac{\Psi(L(t, r))}{L(t, r)}\right)^{\frac{\mu}{\mu-1}} d t d r\right)^{\frac{\mu-1}{\mu}},
$$

and $D_{1} u(s, \vartheta)=(\partial / \partial s) u(s, \vartheta), D_{2} u(s, \vartheta)=(\partial / \partial t) u(s, \vartheta), D_{2} D_{1} u(s, \vartheta)=D_{2} D_{1} u(s, \vartheta)=$ $\left(\partial^{2} / \partial s \partial \vartheta\right) u(s, \vartheta)$.

\section{Conclusions}

In the context of this article, we presented generalizations of symmetric Hilbert-type inequalities on time scales. Our consequences are considered in rather general forms and contain several special integral and discrete of symmetric inequalities. The technique is based on the applications of well-known inequalities and new tools from time scale calculus, which is used in various problems involving symmetry for Hilbert-type inequalities. For future work, we can present such inequalities by using Riemann-Liouville type fractional integrals and fractional derivatives on time scales, which has many applications of symmetric and asymmetric Hilbert-type inequalities. It will also be very interesting to present such inequalities on quantum calculus. 
Author Contributions: Conceptualization, M.Z., H.A.A.E.-H. and H.M.R.; Data curation, P.A., M.Z., and H.M.R.; Formal analysis, G.A.;Funding acquisition, M.Z.; Investigation, G.A., H.A.A.E.-H. and P.A.; Methodology, G.A. and H.A.A.E.-H.; Visualization, H.M.R.; Writing—original draft, M.Z. and H.M.R.; Writing-review \& editing, M.Z., H.A.A.E.-H. and P.A. All authors have read and agreed to the published version of the manuscript.

Funding: This research was funded by the Deanship of Scientific Research at Princess Nourah Bint Abdulrahman University through the Fast-track Research Funding Program.

Acknowledgments: This research was funded by the Deanship of Scientific Research at Princess Nourah Bint Abdulrahman University through the Fast-track Research Funding Program.

Conflicts of Interest: The authors declare that they have no competing interests.

\section{References}

1. Hardy, G.H.; Littlewood, J.E.; Pólya, G. Inequalities, 2nd ed.; Cambridge University Press: Cambridge, UK, 1934.

2. Abd El-Hamid, H.A.; Rezk, H.M.; Ahmed, A.M.; AlNemer, G.; Zakarya, M.; El Saify, H.A. Dynamic Inequalities in Quotients with General Kernels and Measures. J. Funct. Spaces 2020, 2020, 5417084.

3. Saker, S.H.; Kenawy, M.R.; AlNemer, G.; Zakarya, M. Some Fractional Dynamic Inequalities of Hardys Type Via Conformable Calculus. Mathematics 2020, 8, 434. [CrossRef]

4. Cheung, W.-S.; Hanjš, Ž.; Pečarić, J. Some Hardy-type inequalities. J. Math. Anal. Appl. 2000, 250, 621-634. [CrossRef]

5. Gao, M.; Yang, B. On the extended Hilbert's inequality. Proc. Am. Math. Soc. 1998, 126, 751-759.

6. Jichang, K. On New Extensions of Hilbert's Integral Inequality. J. Math. Anal. Appl. 1999, 235, 608-614. [CrossRef]

7. Handley, G.D.; Koliha, J.J.; Pečarić, J.E. New Hilbert-Pachpatte type integral inequalities. J. Math. Anal. Appl. 2001, 257, 238-250. [CrossRef]

8. Pachpatte, B.G. On some new inequalities similar to Hilbert's inequality. J. Math. Anal. Appl. 1998, 226, 166-179. [CrossRef]

9. Yang, B. On new generalizations of Hilbert's inequality. J. Math. Anal. Appl. 2000, 248, 29-40.

10. Handley, G.D.; Koliha,J.J.; Pečarić, J.E. A Hilbert type inequality. Tamkang J. Math. 2000, 31, 311-315.

11. Yang, B.; Rassias, T.M. On the way of weight coefficient and research for the Hilbert-type inequalities. Math. Appl. 2003, 6, 625-658. [CrossRef]

12. Zhao, C.-J. Generalization on two new Hilbert type inequalities. J. Math. 2000, 20, 413-416.

13. Pachpatte, B.G. A note on Hilbert type inequality. Tamkang J. Math. 1998, 29, 293-298.

14. Pachpatte, B.G. Inequalities Similar to Certain Extensions of Hilbert's Inequality. J. Math. Anal. Appl. 2000, 243, 217-227. [CrossRef]

15. Kim, Y.H.; Kim, B.I. An Analogue of Hilbert's inequality and its extensions. Bull. Korean Math. Soc. 2002, 39, 377-388. [CrossRef]

16. Hilger, S. Ein Maßkettenkalkül mit Anwendung auf Zentrumsmannigfaltigkeiten. Ph.D. Thesis, Universität Würzburg, Würzburg, Germany, 1988.

17. O’Regan, D.; Rezk, H.M.; Saker, S.H. Some Dynamic Inequalities Involving Hilbert and Hardy-Hilbert Operators with Kernels. Results Math. 2018, 73, 146. [CrossRef]

18. Saker, S.H.; Ahmed, A.M.; Rezk, H.M.; O'Regan, D.; Agarwal, R.P. New Hilbert's dynamic inequalities on time scales. Math. Inequalities Appl. 2017, 20, 1017-1039. [CrossRef]

19. Saker, S.H.; Rezk, H.M.; Abohela, I.; Baleanu, D. Refinement multidimensional dynamic inequalities with general kernels and measures. J. Inequalities Appl. 2019, 2019, 306. [CrossRef]

20. Saker, S.H.; Rezk, H.M.; O’Regan, D.; Agarwal, R.P. A variety of inverse Hilbert type inequality on time scales. Dyn. Contin. Discret. Impuls. Syst. Ser. A Math. Anal. 2017, 24, 347-373.

21. Saker, S.; Rezk, H.M.; Krnić, M. More accurate dynamic Hardy-type inequalities obtained via superquadraticity. Rev. Real Acad. Cienc. Exactas Fis. Nat. Ser. A Mat. 2019, 113, 2691-2713. [CrossRef]

22. Bohner, M.; Peterson, A. Dynamic Equations on Time Scales: An Introduction with Applications; Birkhäuser: Boston, MA, USA, 2001.

23. Bohner, M.; Peterson, A. (Eds.) Advances in Dynamic Equations on Time Scales; Birkhäuser: Boston, MA, USA, 2003. 
24. Agarwal, R.; O’Regan, D.; Saker, S.H. Dynamic Inequalities on Time Scales; Springer International Publishing: Cham, Switzerland, 2014.

25. Ozkan, U.M.; Yildirim, H. Hardy-Knopp-type inequalities on time scales. Dyn. Syst. Appl. 2008, 17, 477-486.

26. Bibi, R.; Bohner, M.; Pečarić, J.; Varosanec, S. Minkowski and Beckenbach-Dresher inequalities and functionals on time scales. J. Math. Inequalities 2013, 7, 299-312. [CrossRef]

27. Mitrinovic, D.S.; Pecaric, J.E.; Fink, A.M. Classical and New Inequalities in Analysis; Kluwer Academic: Dordrech, The Netherlands, 1993.

28. Ahmed, A.M.; AlNemer, G.; Zakarya, M.; Rezk, H.M. Some Dynamic Inequalities of Hilbert's Type. J. Funct. Spaces 2020, 2020, 4976050. [CrossRef]

29. Saker, S.H.; El-Deeb, A.A.; Rezk, H.M.; Agarwal, R.P. On Hilbert's inequality on time scales. Appl. Anal. Discret. Math. 2017, 11, 399-423. [CrossRef]

(C) 2020 by the authors. Licensee MDPI, Basel, Switzerland. This article is an open access article distributed under the terms and conditions of the Creative Commons Attribution (CC BY) license (http://creativecommons.org/licenses/by/4.0/). 